\title{
Necitumumab plus platinum-based chemotherapy versus chemotherapy alone as first-line treatment for stage IV non-small cell lung cancer: a meta-analysis based on randomized controlled trials
}

\author{
Li Wang ${ }^{1,2}$, Chen Liao ${ }^{1,2}$, Meng $\mathrm{Li}^{2,3}$, Shujuan Zhang ${ }^{2,3}$, Fengming $\mathrm{Yi}^{3}$, Yiping Wei ${ }^{1}$, Jiao Yu ${ }^{1}$, \\ Wenxiong Zhang ${ }^{1}$
}

${ }^{1}$ Department of Thoracic Surgery, the Second Affiliated Hospital of Nanchang University, Nanchang, China; ${ }^{2}$ Jiangxi Medical College, Nanchang University, Nanchang, China; ${ }^{3}$ Department of Oncology, the Second Affiliated Hospital of Nanchang University, Nanchang, China

Contributions: (I) Conception and design: L Wang, W Zhang; (II) Administrative support: W Zhang, F Yi, Y Wei, J Yu; (III) Provision of study materials or patients: L Wang, F Yi, Y Wei; (IV) Collection and assembly of data: L Wang, C Liao, M Li, S Zhang; (V) Data analysis and interpretation: L Wang, C Liao, M Li, S Zhang; (VI) Manuscript writing: All authors; (VII) Final approval of manuscript: All authors.

Correspondence to: Wenxiong Zhang, MD. Department of Thoracic Surgery, the Second Affiliated Hospital of Nanchang University, 1 Minde Road, Nanchang, China. Email: zwx123dr@126.com.

\begin{abstract}
Background: Whether necitumumab combined with platinum-based chemotherapy (NC) for treating stage IV non-small cell lung cancer (NSCLC) as a first-line treatment could enhance antitumor effectiveness compared with platinum-based chemotherapy alone (CA) treatment is still controversial. The antitumor effectiveness and toxicity of the two treatments were compared in this meta-analysis.

Methods: We searched in PubMed, ScienceDirect, Scopus, Web of Science, Ovid MEDLINE, the Cochrane Library, Embase, and Google Scholar to acquire applicable articles. The outcome indicators mainly included progression-free survival (PFS), overall survival (OS) and adverse effects (AEs).

Results: Eight articles based on 4 randomized controlled trials were obtained. The NC group had a longer PFS [95\% confidence interval (CI): 0.84-0.99, $\mathrm{P}=0.03$ ] and a higher disease control rate (DCR, 95\% CI: $1.01-1.10, \mathrm{P}=0.03$ ) than those of the CA group. OS (95\% CI: $0.85-1.01, \mathrm{P}=0.09$ ) and the objective response rate (ORR, 95\% CI: 0.93-1.71, P=0.14) were similar in the NC and CA groups. Nevertheless, in both quantity and extent, the NC treatment had more severe skin rash, hypomagnesemia, and venous thromboembolism than those of the CA treatment. Subanalysis suggested that the advantage of OS was more obvious in the NC group than that in the CA group in patients with high epidermal growth factor receptor (EGFR) expression.

Conclusions: With a longer PFS and a higher DCR, NC treatment seemed to be more suitable for treating stage IV NSCLC as first-line therapy, especially for those with high EGFR expression, but its AEs could not be ignored.
\end{abstract}

Keywords: Necitumumab; platinum; chemotherapy; stage IV; non-small cell lung cancer (NSCLC); meta-analysis

Submitted Oct 13, 2019. Accepted for publication Aug 19, 2020.

doi: 10.21037/apm-19-365

View this article at: http://dx.doi.org/10.21037/apm-19-365 


\section{Introduction}

Regardless of sex, lung carcinoma is the second most common cancer (1), and it accounts for the largest proportion of cancer deaths (accounting for $18.4 \%$ of the total), which imposes a heavy worldwide burden (2). Because early disease is typically asymptomatic, the majority of nonsmall cell lung cancer (NSCLC) is not diagnosed until stage III or IV (3). Surgical treatment and radiotherapy are not suitable for stage IV NSCLC as the first-line treatment, so the platinum-based chemotherapy alone (CA) treatment has long been the standard preferred treatment of NSCLC at stage IV (4,5). Nevertheless, the efficacy of CA treatment was considered to have reached a treatment bottleneck $(6,7)$. The application of adding epidermal growth factor receptor (EGFR) antibody to multiple platinum-based chemotherapy regimens is a hotspot in the clinic, especially for treating NSCLC (8).

Necitumumab is one of the second-generation EGFR antibodies. Due to the binding of the receptor to the ligand, it competes with EGFR to prevent receptor activation and downstream signaling $(9,10)$. Currently, adding necitumumab to platinum-based chemotherapy for treating stage IV NSCLC as first-line treatment is a hotspot in the clinic. Recently, with the permission of the US Food and Drug Administration, necitumumab combined with gemcitabine and cisplatin chemotherapy has been the firstline therapy for squamous NSCLC that has metastasized (11). Nevertheless, it is still controversial whether NC treatment has a better antitumor effect than CA treatment. Thatcher's study of the SQUIRE trial showed better antitumor effectiveness with necitumumab added to the platinumbased chemotherapy alone group (12). However, NC treatment was considered to have a higher incidence of skin rash, venous thromboembolism, eye disorders, hypomagnesemia and dose discontinuation related to total adverse events (AEs) than those of CA treatment $(13,14)$. In Paz-Ares's study of the INSPIRE trial and Spigel's phase II randomized controlled trial (RCT), it was found that progression-free survival (PFS), overall survival (OS), objective response rate (ORR), and disease control rate (DCR) were similar between the NC arm and CA arm $(15,16)$.

To resolve the controversy, we included all relevant articles to conduct this meta-analysis, with the OS, PFS, ORR, DCR, all grade AEs and grade 3-5 AEs compared between NC treatment and CA treatment for treating stage IV NSCLC as first-line treatment. We present the following article in accordance with the PRISMA reporting checklist (available at http://dx.doi.org/10.21037/apm-19-365).

\section{Methods}

This meta-analysis was based on PRISMA (Preferred Reporting Items for Systematic Review and Meta-Analysis) guidelines.

\section{Protocol and registration}

We have registered the review protocol in the PROSPERO, which can be accessed by visiting the https://www.crd.york. ac.uk/PROSPERO. And our Systematic review registration number is CRD42020147170.

\section{Search strategy}

We searched in PubMed, ScienceDirect, The Cochrane Library, Scopus, Web of Science, EMBASE, Ovid MEDLINE and Google Scholar. The last search to select relevant articles comparing NC treatment with CA treatment as first-line treatment for stage IV NSCLC was conducted on May 20, 2019. We used "necitumumab", "lung cancer" and "chemotherapy". Details of the retrieval are accessible in Supplementary file. We also searched further eligible articles using the references of retrieved articles.

\section{Selection criteria}

Our included studies adhered to PICOS principles, and the details were as follows: (I) P (patients): patients with NSCLC were at stage IV histologically or cytologically (on the basis of the AJCC 7th edition) (17); (II) I (interference) vs. C (comparison): NC vs. CA as first-line treatment; (III) O (outcomes): outcomes were OS, PFS, OSR, PFSR, ORR, DCR and AEs; (IV) $\mathrm{S}$ (study design): RCTs published in English.

Those articles without initial data, meta-analyses, conference articles, case reports, and articles from the same experimental center on the same topic were excluded.

\section{Data extraction}

Data regarding the authors, clinical trials center, timeline, number of participants in two groups, research design, patients' baseline data (age, sex, study period, pretreatment), antitumor efficacy indicators [PFS, OS, ORR, DCR, progression-free survival rate (PFSR) and overall survival 
rate (OSR)] and the number of all grade AEs as well as grade 3-5 AEs were gathered by two investigators independently. The disagreements under various circumstances were settled by a third researcher.

We mainly used OS, PFS, OSR, and PFSR to analyze survival dates. For OSR and PFSR, we divided the analysis into six months and analyzed them for a total of two years. And the subgroup analysis of OS and PFS were estimated based on the EGFR mutation status, sex, age, region, pathology and treatment.

\section{Quality assessment}

The 5-point Jadad scale and Cochrane Risk Assessment Tool were used to evaluate the quality of RCTs. The Jadad scale mainly includes the following three aspects: randomizing, blinding, and including all patients. A study is regarded as high quality if it receives a score of $\geq 3$ points (18).

The Cochrane Risk Assessment Tool mainly focuses on the bias of selection, performance, detection, attrition, reporting and others, the risk of which was assessed using low, unclear and high risk, respectively (19). Then, the results are presented as the risk of bias graph.

Grading of Recommendations Assessment, Development and Evaluation (GRADE) is used to evaluate the level of evidence of included studies. GRADE mainly focuses on the risk of bias, discordance, indirectness, inaccuracy and publication bias, among which the results included four levels: high, medium, low and very low (20).

\section{Statistical analysis}

The accomplishment of this meta-analysis was based on ReMan5.3 and STATA 12.0. We used hazard ratios (HRs) to analyze PFS and OS. Kaplan-Meier curves provided HRs and 95\% CIs. We acquired OSR and PFSR from the Kaplan-Meier curves directly. We analyzed PFS and OS using HRs (HR <1 favors NC treatment) and 95\% CIs. The ORR, DCR, PFSR, OSR (RR >1 favors NC treatment) and AEs (RR $<1$ favors NC treatment) were analyzed through risk ratios (RRs) and $95 \%$ CIs. Subanalysis of PFS and OS was executed to test whether these outcome indicators would change in accordance with the EGFR expression situation. The $\chi^{2}$ test with $\mathrm{I}^{2}$ statistic was applied to assess heterogeneity. We would choose the random-effects model if $\mathrm{I}^{2}>50 \%$ or $\mathrm{P}<0.1$, which suggested serious heterogeneity; otherwise, we would prefer the fixed-effects model. We conducted sensitivity analyses of PFS, OS, ORR, and
DCR. We used Begg's test and Egger's test to evaluate the publication bias, among which $\mathrm{P}<0.05$ suggested statistical significance.

\section{Results}

\section{Search results and quality assessment}

As shown in Figure 1, 8 articles based on 4 RCTs involving 2,074 patients (NC group, 1,060; CA group, 1,014) were selected for this meta-analysis (5,12,14-16,21-23). Five articles were from the phase III SQUIRE trial, among which Reck's study analyzed the tolerability and quality of life (21), Paz-Ares L's study analyzed the safety and efficacy outcomes of EGFR-expression patients (5), Reck's other study paid more attention to a German subgroup (22), PazAres L's other study focused on east Asian patients, and one focused on the anticancer effectiveness based on OS, PFS, ORR, and DCR with toxicity based on AEs (15). The indicators they analyzed were different, so we analyzed several articles. The other three articles came from three different RCTs.

All 8 articles scored 4-5 points and were of high quality. The baseline information and major assessment index of all contained articles are listed in Table 1. The GRADE results showed that all 8 articles had high or medium quality, and more details are presented in Table S1. All 4 studies judged by Cochrane Risk Assessment were mostly at low risk, and more details are shown in Figure S1.

\section{Anticancer effectiveness}

Four articles made a comparison of OS (heterogeneity: $\left.\mathrm{P}=0.68, \mathrm{I}^{2}=0 \%\right)$. The $\mathrm{OS}$ results tended to favor $\mathrm{NC}$ treatment $(\mathrm{HR}=0.93$, 95\% CI: $0.85-1.01, \mathrm{P}=0.09$; Figure $2 A)$, OSR-0.5y (RR =1.04, 95\% CI: 0.94-1.16, P=0.43), OSR-1y ( $\mathrm{RR}=1.13$, 95\% CI: 0.94-1.36, $\mathrm{P}=0.20)$ and OSR-1.5y (RR $=1.07,95 \% \mathrm{CI}: 0.92-1.25, \mathrm{P}=0.39)$ and OSR-2y $(\mathrm{RR}=1.24$, 95\% CI: 0.70-2.17, P=0.46) (Figure S2). And it demonstrated that the difference of OSR between the two groups tended to increase firstly, then to reach a maximum after following up one year and finally to reduce (Figure S3A).

Four articles made a comparison of PFS (heterogeneity: $\mathrm{P}=0.27, \mathrm{I}^{2}=24 \%$ ), where the NC group had a longer PFS (HR $=0.91,95 \%$ CI: 0.84-0.99, $\mathrm{P}=0.03$; Figure $2 B)$. And it was shown that with time going by, the discrepancy of PFSR between two groups went smaller and smaller (Figure S3B). There were no significant differences in PFSR-0.5y (RR 


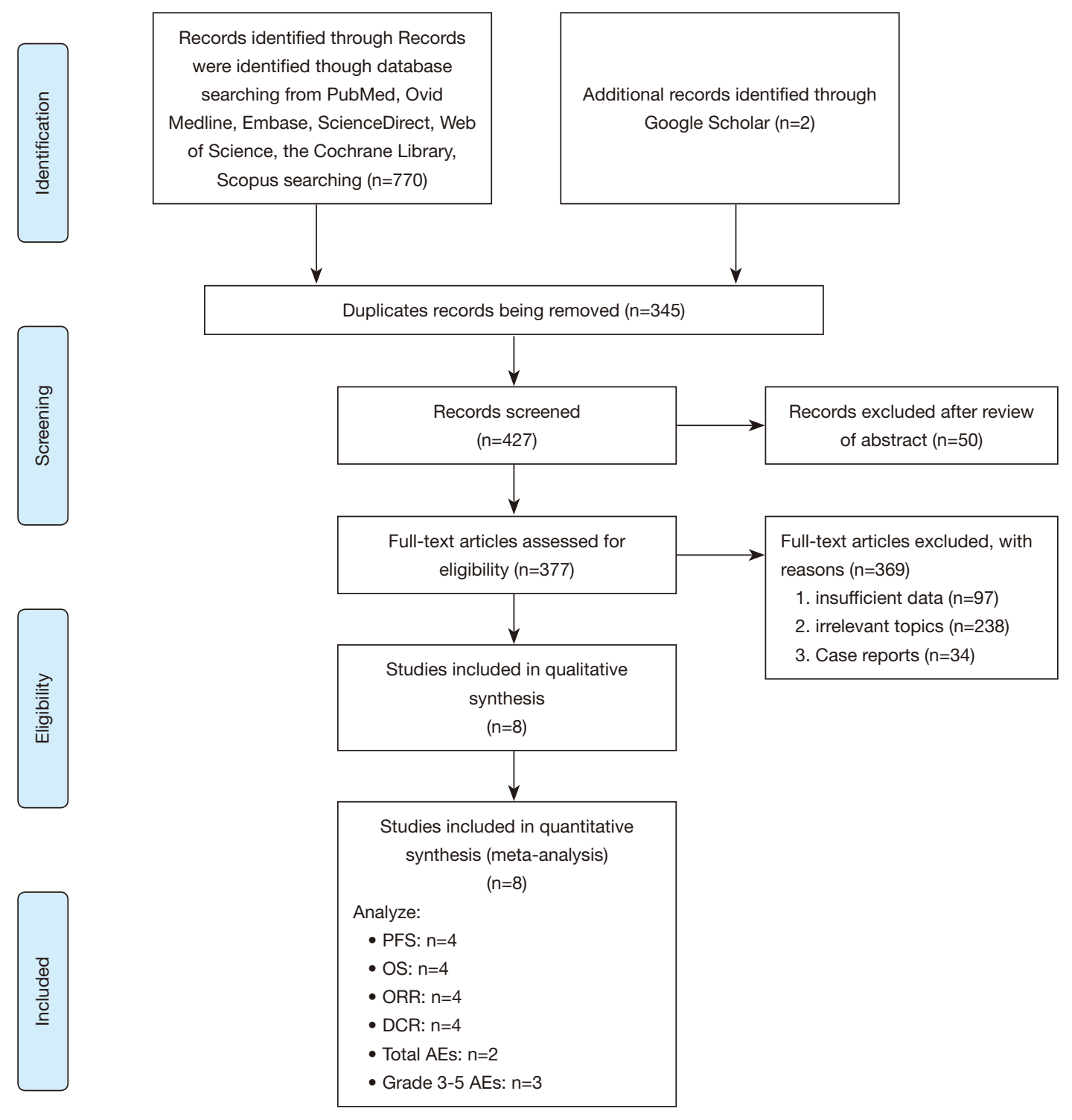

Figure 1 Flow chart of study selection.

$=1.22,95 \%$ CI: 0.88-1.69, P=0.23), PFSR-1y $(\mathrm{RR}=1.28$, 95\% CI: 0.95-1.72, P=0.11), PFSR-1.5y $(\mathrm{RR}=1.34$, 95\% CI: 0.84-2.15, $\mathrm{P}=0.22)$ and PFSR-2y $(\mathrm{RR}=2.01,95 \%$ CI: $0.82-4.96, \mathrm{P}=0.13$ ) (Figure S4).

Four articles made a comparison of ORR (heterogeneity: $\mathrm{P}=0.004, \mathrm{I}^{2}=77 \%$ ). Obvious differences were not observed between the treatments $(\mathrm{RR}=1.26,95 \% \mathrm{CI}$ : 0.93-1.71, $\mathrm{P}=0.14$; Figure 3 A). Four articles made a comparison of DCR (heterogeneity: $\mathrm{P}=0.27, \mathrm{I}^{2}=24 \%$ ). The $\mathrm{NC}$ group had a high DCR (RR $=1.05,95 \%$ CI: 1.01-1.10, $\mathrm{P}=0.03$; Figure 3B). The subgroup analysis showed that CA treatment was related to a higher rate of complete remission (CR)
$(\mathrm{RR}=0.14$, 95\% CI: 0.02-0.79, P=0.03; Figure S5A) and an obvious difference was not discovered between both groups for partial remission (PR) $(\mathrm{RR}=1.29$, 95\% CI: 0.96-1.74, $\mathrm{P}=0.09$; Figure $\mathrm{S} 5 \mathrm{~B})$ and stable disease $(\mathrm{SD})(\mathrm{RR}=0.95$, 95\% CI: 0.81-1.11, P=0.52; Figure S5C). However, to our surprise, CA treatment was also related to a higher rate of progression of disease (PD) $(\mathrm{RR}=0.68,95 \% \mathrm{CI}: 0.53-0.89$, $\mathrm{P}=0.005$; Figure S5D).

\section{Toxicity}

We compared the toxicity between the NC group and CA 
Table 1 Characteristics of included studies

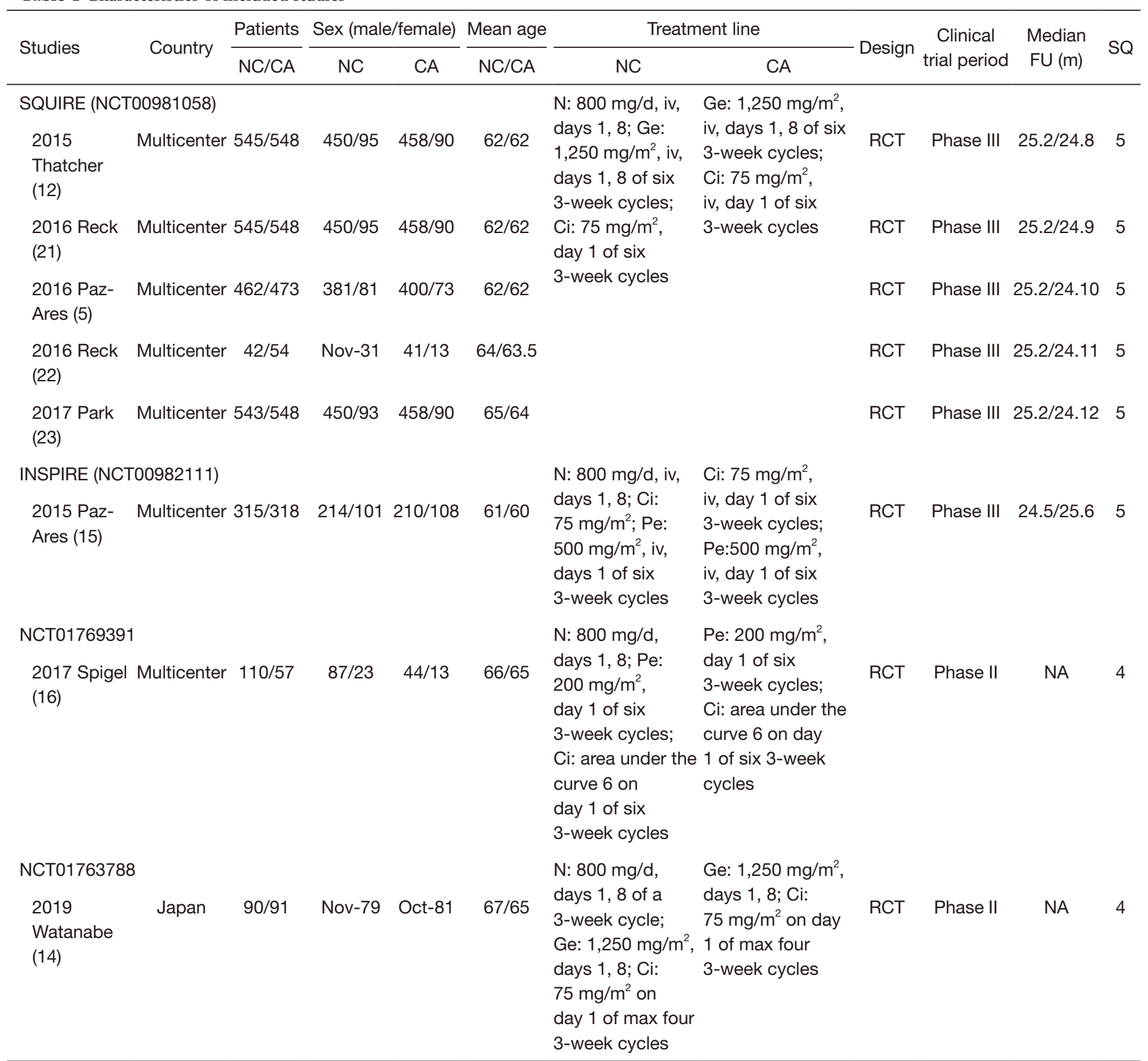

NC, necitumumab plus platinum-based chemotherapy; CA, platinum-based chemotherapy alone; N, necitumumab; Ge, gemcitabine; Ci, cisplatin; Pe, pemetrexed; FU, follow up; SQ, score quality; RCT, randomized controlled trail; NA, not available.

group regarding all grade AEs, grade 3-5 $\mathrm{AEs}$ and the 10 most reported AEs.

Two articles compared all grade AEs (heterogeneity: $\left.\mathrm{P}=0.05, \mathrm{I}^{2}=74 \%\right)$. Distinct differences were not observed between the treatments (95\% CI: 0.97-1.31, P=0.13; Figure $4 A$ ). The 10 most reported all grade AEs were skin rash, hypomagnesemia, hypersensitivity, eye disorders, arterial thromboembolism, venous thromboembolism, anemia, neutropenia, fatigue, and thrombocytopenia, and NC treatment had a higher risk of skin rash, venous thromboembolism, eye disorders and hypomagnesemia than that of the CA group. More details are shown in Table 2.

Grade 3-5 AEs were compared in three studies (heterogeneity: $\mathrm{P}=0.16, \mathrm{I}^{2}=45 \%$ ), and $\mathrm{CA}$ treatment was at 
A

\begin{tabular}{|c|c|c|c|c|c|c|c|}
\hline Study or Subgroup & log[Hazard Ratio] & $\mathrm{SE}$ & Weight & $\begin{array}{l}\text { Hazard Ratio } \\
\text { IV. Fixed. } 95 \% \mathrm{Cl}\end{array}$ & $\begin{array}{l}\text { Hazard } \\
\text { IV. Fixed }\end{array}$ & $\begin{array}{l}\text { d Ratio } \\
\text { d. } 95 \% \mathrm{Cl}\end{array}$ & \\
\hline Watanabe 2019 & -0.18 & 0.117 & $14.0 \%$ & $0.84[0.66,1.05]$ & 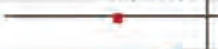 & $F_{-}$ & \\
\hline Spigel 2017 & -0.081 & 0.247 & $3.1 \%$ & $0.92[0.57,1.50]$ & & & \\
\hline Paz-Ares 2015 & -0.076 & 0.056 & $61.1 \%$ & $0.93[0.83,1.03]$ & $\rightarrow$ & & \\
\hline Thatcher 2015 & 0.004 & 0.094 & $21.7 \%$ & $1.00[0.84,1.21]$ & & & \\
\hline Total $(95 \% \mathrm{Cl})$ & & & $100.0 \%$ & $0.93[0.85,1.01]$ & & & \\
\hline \multicolumn{5}{|c|}{$\begin{array}{l}\text { Heterogeneity: } \mathrm{Ch}^{2}=1.51, d f=3(P=0.68) ; I^{2}=0 \% \\
\text { Test for overall effect: } Z=1.68(P=0.09)\end{array}$} & $0.7 \underset{1}{0.85}{ }^{1}$ & $\begin{array}{c}1.2 \\
1 \\
\text { Favours CA }\end{array}$ & 1.5 \\
\hline
\end{tabular}

B

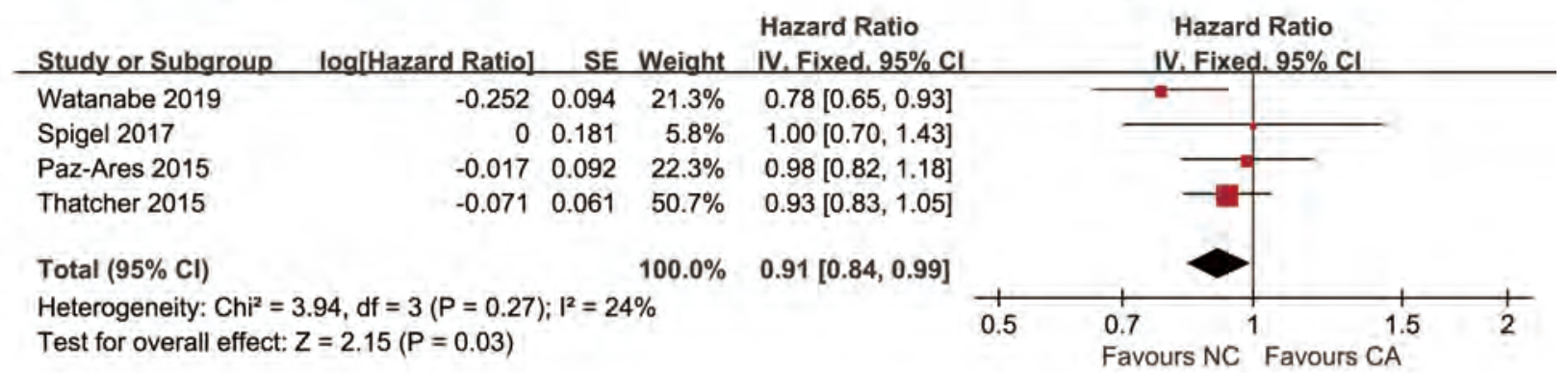

Figure 2 Forest plot of the HRs of OS (A) and PFS (B) associated with necitumumab plus platinum-based chemotherapy versus platinumbased chemotherapy alone. HRs, hazard ratios; OS, overall survival; PFS, progression-free survival.

A

\begin{tabular}{|c|c|c|c|c|c|c|c|c|}
\hline Study or Subgroup & $\begin{array}{l}\text { NC } \\
\text { Events }\end{array}$ & Total & $\begin{array}{l}\text { CA } \\
\text { Events }\end{array}$ & Total & Weight & $\begin{array}{l}\text { Risk Ratio } \\
\text { M-H. Random, } 95 \% \mathrm{Cl}\end{array}$ & & $\begin{array}{l}\text { Risk Ratio } \\
\text { M-H. Random, } 95 \% \mathrm{Cl}\end{array}$ \\
\hline Watanabe 2019 & 46 & 90 & 19 & 91 & $19.7 \%$ & $2.45[1.56,3.83]$ & & \\
\hline Spigel 2017 & 46 & 110 & 20 & 57 & $20.9 \%$ & $1.19[0.79,1.81]$ & & \\
\hline Paz-Ares 2015 & 99 & 315 & 102 & 318 & $28.8 \%$ & $0.98[0.78,1.23]$ & & \\
\hline Thatcher 2015 & 170 & 545 & 158 & 548 & $30.6 \%$ & $1.08[0.90,1.30]$ & & \\
\hline Total $(95 \% \mathrm{Cl})$ & & 1060 & & 1014 & $100.0 \%$ & $1.26[0.93,1.71]$ & & \\
\hline Total events & 361 & & 299 & & & & & \\
\hline $\begin{array}{l}\text { Heterogeneity: } \mathrm{Tau}^{2}= \\
\text { Test for overall effect: }\end{array}$ & $\begin{array}{l}.07 ; \mathrm{Ch}^{2} \\
z=1.49(\mathrm{~F}\end{array}$ & $\begin{array}{l}=13.23 \\
P=0.14\end{array}$ & $d f=3($ & $P=0.0$ & $04) ; 1^{2}=7$ & & 0.2 & $\begin{array}{ccc}0.5 & 1 & 2 \\
\text { Favours CA } & 2 \\
\text { Favours NC }\end{array}$ \\
\hline
\end{tabular}

B

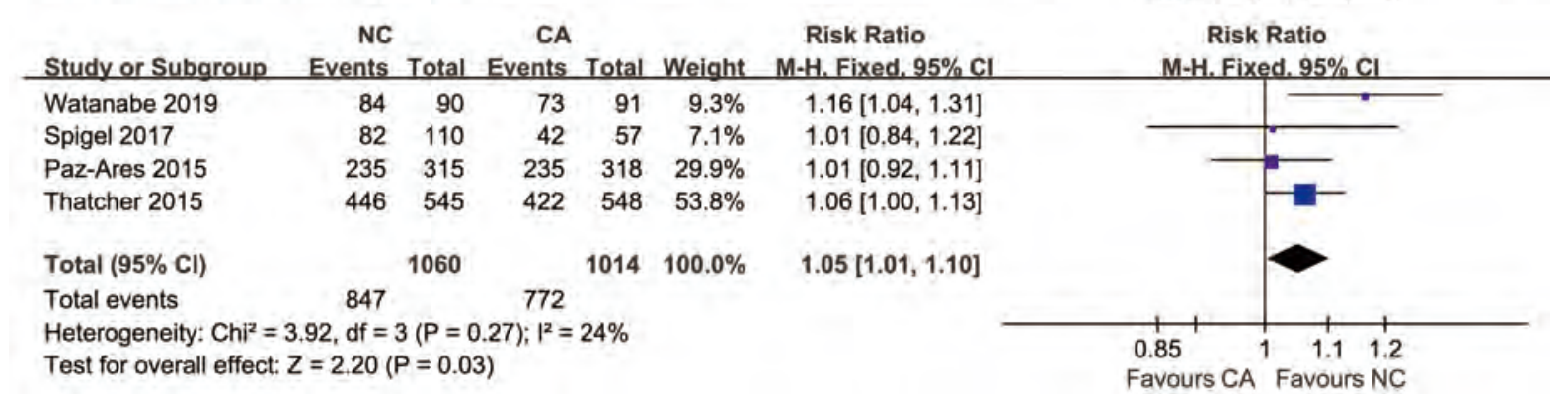

Figure 3 Forest plots of the ORR (A) and DCR (B) associated with necitumumab plus platinum-based chemotherapy versus platinum-based chemotherapy alone. ORR, objective response rate; DCR, disease control rate.

a lower risk of grade 3-5 AEs than NC treatment (95\% CI: $1.08-1.25, \mathrm{P}<0.0001$; Figure $4 B)$. The ten most reported grade 3-5 AEs were skin rash, hypomagnesemia, arterial thromboembolism, venous thromboembolism, anemia, neutropenia, fatigue, thrombocytopenia, febrile neutropenia and diarrhea, and $\mathrm{NC}$ treatment was at higher risks of skin 
A

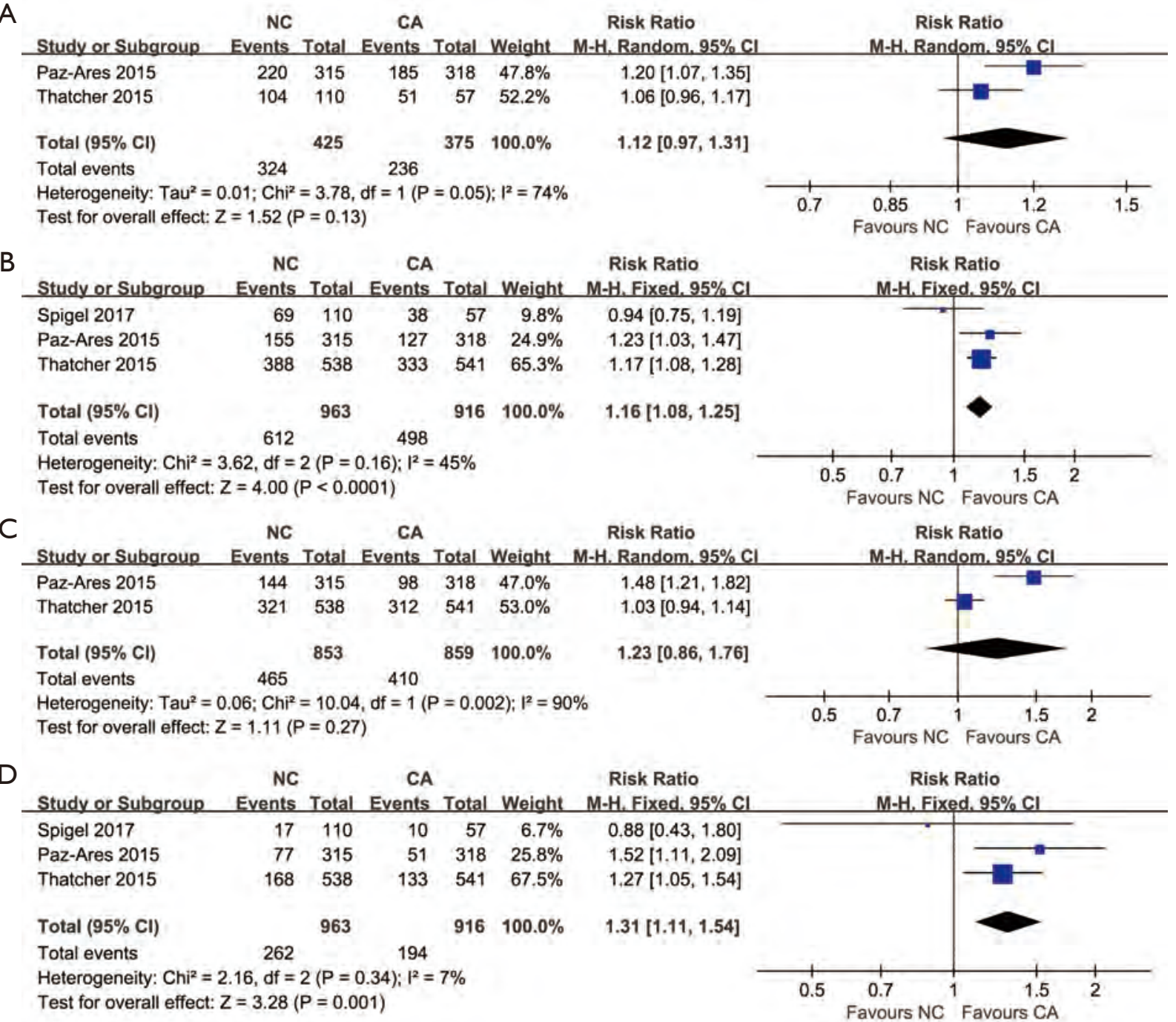

Figure 4 Forest plots of the RRs of total AEs (A), grade 3-4 AEs (B), drug reductions (C) and drug discontinuations (D) associated with necitumumab plus platinum-based chemotherapy versus platinum-based chemotherapy alone. RR, risk ratios; AEs, adverse effects.

rash, hypomagnesemia and venous thromboembolism than was the CA group. More details are shown in Table 3.

Some people suffered dose reduction or discontinuation due to severe AEs. Two studies compared dose reduction (heterogeneity: $\mathrm{P}=0.002, \mathrm{I}^{2}=90 \%$ ). Obvious differences were not observed between the treatments (95\% CI: 0.86-1.76, $\mathrm{P}=0.27$; Figure $4 C$ ). Three studies compared dose discontinuation (heterogeneity: $\mathrm{P}=0.34, \mathrm{I}^{2}=7 \%$ ). The $\mathrm{NC}$ treatment seemed to be more likely to result in dose discontinuation (95\% CI: 1.11-1.54, P=0.001; Figure 4D).

\section{Subgroup analysis}

To determine whether the antitumor effectiveness of NC treatment compared with CA treatment would be different in subgroups, the pooled results of OS and PFS were estimated based on the EGFR mutation status, sex, age, region, pathology and treatment (Table 4).

The pooled results of the selected articles found that in the EGFR mutation status subgroup, the OS for $\mathrm{NC}$ treatment versus CA treatment was more favorable in those with high EGFR expression rather than low EGFR 
Table 2 Top 10 adverse effects (all grade) associated with necitumumab plus platinum-based chemotherapy versus platinum-based chemotherapy alone

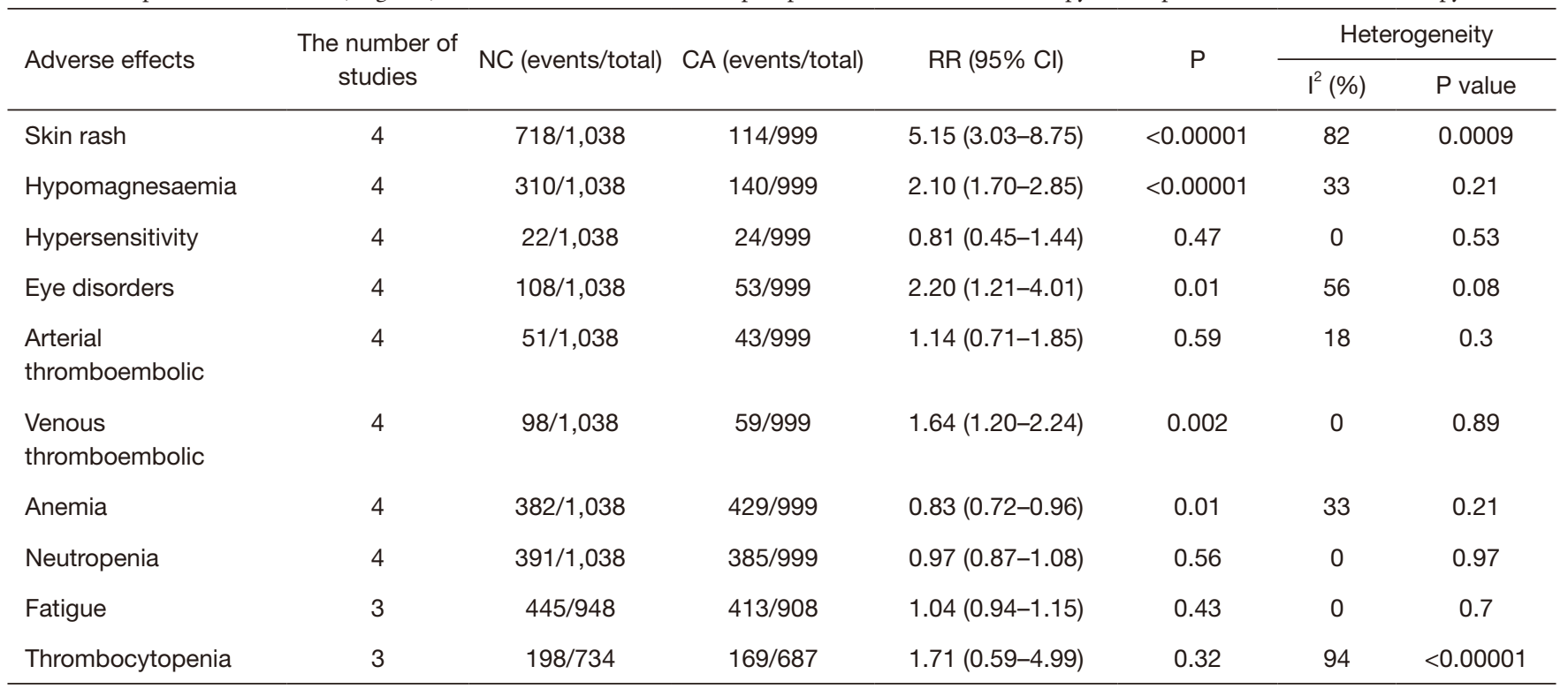

$\mathrm{NC}$, necitumumab plus platinum-based chemotherapy; CA, platinum-based chemotherapy alone; RR, risk ratio; $\mathrm{Cl}$, confidence interval.

Table 3 Top 10 adverse effects (3-4 grade) associated with necitumumab plus platinum-based chemotherapy versus platinum-based chemotherapy alone

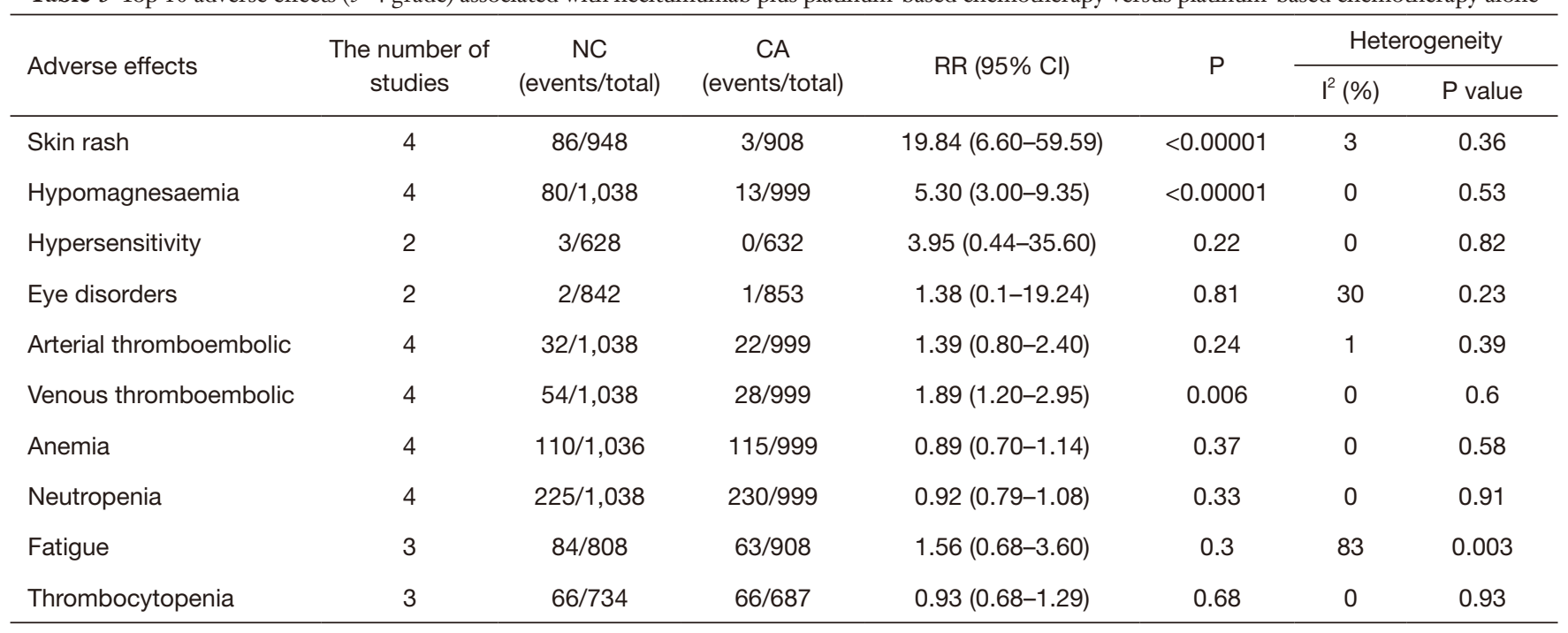

$\mathrm{NC}$, necitumumab plus platinum-based chemotherapy; CA, platinum-based chemotherapy alone; RR, risk ratio; Cl, confidence interval.

expression (HR: 0.75, 95\% CI: 0.60-0.94 vs. HR: 0.90, 95\% CI: $0.75-1.07)$. It was also found in the pathology subgroup that the HRs for OS (HR: 0.89, 95\% CI: 0.80-0.98, $\mathrm{P}=0.02$ ) and PFS (HR: 0.89, 95\% CI: 0.81-0.98, $\mathrm{P}=0.02$ ) for NC therapy were more favorable in squamous NSCLC. Females had a longer PFS. Patients under 70 years old had a longer OS and PFS. Caucasians had a longer OS and PFS.
Obvious differences were not found in other subgroups regarding PFS and OS between the NC and CA treatments.

\section{Sensitivity analysis}

When analyzing ORR, total AEs, as well as grade 3-5 AEs, significant heterogeneity was found. The evaluation of the 
Table 4 Subgroup analysis of OS and PFS of necitumumab plus platinum-based chemotherapy vs platinum-based chemotherapy alone

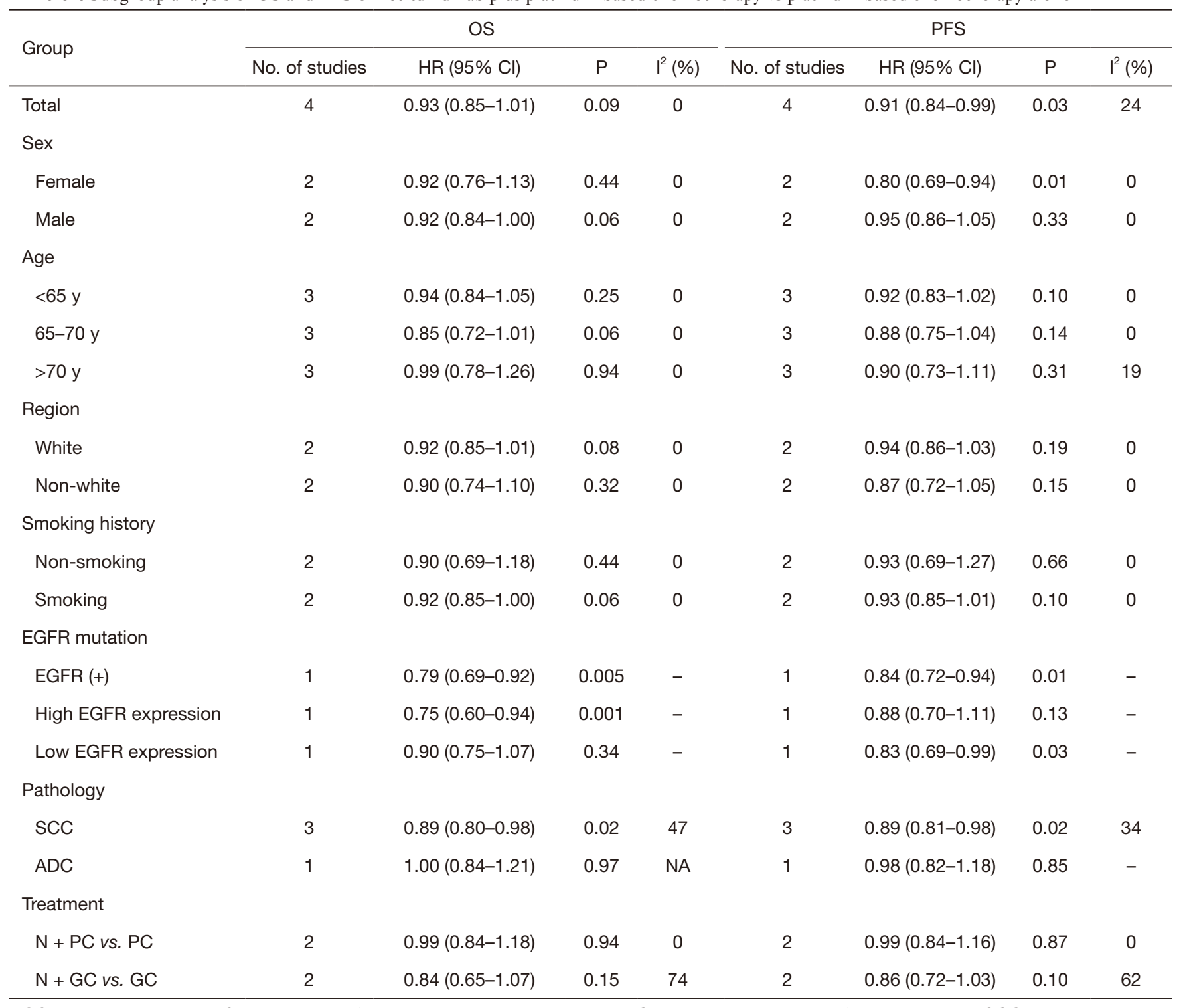

OS, overall survival; PFS, progression-free survival; HR, hazard ratio; EGFR, epidermal growth factor receptor; SCC, squamous cell carcinomas; ADC, adenocarcinoma; N, necitumumab; PC, pemetrexed plus cisplatin; GC, gemcitabine plus cisplatin; NA, not available.

stability and sensitivity was based on the pooled results for the influence of all included studies. The outcomes of PFS (Figure S6A), OS (Figure S6B), ORR (Figure S6C) and DCR (Figure S6D) were reliable and stable.

\section{Publication bias}

Not enough evidence favored publication bias for PFS (Begg's test, $\mathrm{P}=1.000$, Egger's test, $\mathrm{P}=0.977$; Figure $\mathrm{S} 7 \mathrm{~A}$ ), OS (Begg's test, $\mathrm{P}=0.734$, Egger's test, $\mathrm{P}=0.706$; Figure $\mathrm{S} 7 \mathrm{~B}$ ), ORR
(Begg's test, $\mathrm{P}=0.308$; Egger's test, $\mathrm{P}=0.279$; Figure $\mathrm{S} 7 \mathrm{C}$ ) and DCR (Begg's test, $\mathrm{P}=1.000$; Egger's test, $\mathrm{P}=0.949$; Figure S7D).

\section{Discussion}

Most patients are diagnosed at stage IV because early disease is typically asymptomatic (3), and due to a lack of effective surgical treatment and radiotherapy, chemotherapy is widely used as the preferred therapy for those patients 
at stage IV. Whether adding necitumumab to platinumbased chemotherapy for treating stage IV NSCLC as firstline treatment can enhance the antitumor effectiveness is still controversial. As the latest meta-analysis, this study focused on the antitumor effectiveness and toxicity through comparing the $\mathrm{NC}$ group and CA group for stage IV NSCLC as the preferred therapy. Our analysis of 8 articles showed that the NC group had a longer PFS as well as a higher DCR; nevertheless, it was associated with more all-grade skin rash, venous thromboembolism, eye disorders and hypomagnesemia, while grade 3-5 skin rash, hypomagnesemia, and venous thromboembolism in the CA group had a higher rate of CR and PD. The OS results tend to favor NC treatment, there was no obvious difference in PR, and ORR as well as SD were reported in both treatments. In subgroup analysis, the pooled outcomes showed that the NC group may have a longer PFS and OS in those with high EGFR expression as well as in squamous NSCLC.

The importance of antitumor effectiveness when comparing $\mathrm{NC}$ treatment with CA treatment cannot be emphasized enough. Our results indicated that the NC arm had a longer PFS, and there seemed to be a longer OS. It seemed that the advantages of the NC group became more obvious with time. The result of the phase III SQUIRE trial and Spigel's phase II trial showed a clinically meaningful improvement in OS when adding necitumumab to chemotherapy alone $(4,16)$. Watanabe's trial conducted in Japan also showed that adding necitumumab to gemcitabine and cisplatin could lengthen the OS when used as firstline therapy for those NSCLC patients diagnosed at stage IV (14). By contrast, there was no obvious difference in PFS and OS between both treatments when comparing necitumumab combined with gemcitabine and cisplatin versus gemcitabine and cisplatin alone in the phase III INSPIRE trial (15). The reason for the different antitumor effectiveness based on OS between these trials is still unclear. In the EGFR expression subgroup analysis, it was found that compared with the CA group, the $\mathrm{NC}$ group was associated with a longer PFS (HR: 0.85, 95\% CI: 0.74-0.98) and OS (HR: 0.84, 95\% CI: 0.74-0.96); nevertheless, evaluating PFS, it seemed to be similar between the high and low H-score groups [HR: 0.88 (95\% CI: 0.70-1.11) vs. 0.83 (95\% CI: 0.69-0.99)], and it was also reported that the HR for OS for the NC group versus the CA group was more favorable in patients with high EGFR expression rather than low EGFR expression [HR: 0.75 (95\% CI: 0.60-0.94) vs. 0.90 (95\% CI: 0.75-1.07)] (24). Whether high
EGFR expression could be a predictive marker to optimize patients for choosing the NC treatment is still not clear $(25,26)$, so large-scale, high-quality RCTs are needed to answer this question. In the pathology subgroup, the pooled results suggested that NC treatment seemed to be more suitable for squamous NSCLC than CA treatment with a longer OS (HR: 0.89, 95\% CI: 0.80-0.98, $\mathrm{P}=0.02$ ) and PFS (HR: 0.89, 95\% CI: 0.81-0.98, P=0.02). Squamous NSCLC being related to EGFR mutation might account for it (27).

The tumor response rate is another key point when choosing a treatment. Our results showed that the NC treatment had a higher DCR, and no significant difference in ORR between the two treatments was found. It was shown that CA treatment had a higher rate of CR and PD in the subgroup analysis, and no obvious difference was found between the treatments in PR and SD. We tried to use limited studies to explain why the results of the CA group were superior in CR, but what it was that caused this result remained unclear; perhaps large-scale, high-quality studies are required to address this issue. The phase III SQUIRE and INSPIRE trials, in which the patients are from multiple countries, showed that there were no significant differences in ORR and DCR when comparing the two treatments, while an RCT based in Japan suggested a higher rate of ORR and DCR, which might be related to the region where the patients are from, the rate of intravenous infusion or the types of chemotherapy (14). The subgroup analysis results from eastern Asia showed no difference in ORR and DCR (23), which suggested that differences in ORR and DCR resulting in different experiments may not be related to the region where the patients are from. It was also found that although higher clearance and lower exposure were related to body weight, a stimulus test reported that an 800 $\mathrm{mg}$ flat dose could provide optimum response no matter the body weight (28), so the antitumor response rate might not be related to the dose of necitumumab. More large-scale RCTs based on different rates of intravenous infusion and types of chemotherapy are needed to answer this question.

The severe drug toxicity is a controversial problem when choosing the NC treatment. In the analysis, higher incidences of drug reduction, drug discontinuation, skin rash, venous thromboembolism, eye disorders, and hypomagnesemia were found in the NC arm (12-16), which would greatly affect patients' sense of life experience. Reck's study showed that there was no statistical difference, comparing the two treatments, in health-related quality of life, using the Lung Cancer Symptom Scale based on inappetence, exhaustion, coughing, difficulty breathing, 
hemoptysis, ache, normal activity restriction, quality of life and lung cancer symptoms (21). We think that the severe drug toxicity might be related to an additional effect of platinum-based chemotherapy plus necitumumab $(25,29)$. A subgroup analysis of eastern Asia showed that the NC group had a higher incidence of grade 3-5 AEs (23), which suggested that more attention should be paid when eastern Asian patients choose the NC treatment.

Five shortcomings could not be overlooked when taking our conclusions into account. First, the limited number of included articles (only eight) might weaken these results' quality. Second, there was moderately obvious heterogeneity in several comparisons (ORR, dose reduction, all grade AEs, grade 3-5 AEs), which would have an impact on the stability of these results. Third, we just considered those articles published in English with high quality, which might bring up a language bias. Fourth, different pathological types of NSCLC among the contained trials were likely to augment the heterogeneity and lower the quality of the results. Fifth, different combinations and usages of drugs might make a difference in the pooled results.

\section{Conclusions}

Our meta-analysis found the necitumumab plus platinumbased chemotherapy is more effective than chemotherapy alone for stage IV NSCLC as first-line treatment, especially for the EGFR-mutation-positive patients. Nevertheless, the AEs, such as hypomagnesemia, skin rash, venous thromboembolism and eye disorders, that resulted from it should not be overlooked. Additionally, the existing shortcomings of this meta-analysis require extra extensive and high-quality trials to resolve and confirm our conclusion.

\section{Acknowledgments}

The authors thank Professor Bentong Yu, MD (the First Affiliated Hospital of Nanchang University) for her advice and Professor Xiaoshu Cheng, MD, PhD (the Second Affiliated Hospital of Nanchang University) for his data collection. We also appreciate the company of AJE for checking all wordings of the main text, figures and tables. Funding: This study was supported by National Natural Science Foundation of China (NSFC), number of grants (81560345), Natural Science Foundation of Jiangxi Province (grant number: 20181BAB215027). The funding had no role in the design and conduct of the study; collection, management, analysis, and interpretation of the data; preparation, review, or approval of the manuscript; and decision to submit the manuscript for publication.

\section{Footnote}

Reporting Checklist: The authors have completed the PRISMA reporting checklist. Available at http://dx.doi. org/10.21037/apm-19-365

Conflicts of Interest: All authors have completed the ICMJE uniform disclosure form (available at http://dx.doi. org/10.21037/apm-19-365). The authors have no conflicts of interest to declare.

Ethical Statement: The authors are accountable for all aspects of the work in ensuring that questions related to the accuracy or integrity of any part of the work are appropriately investigated and resolved.

Open Access Statement: This is an Open Access article distributed in accordance with the Creative Commons Attribution-NonCommercial-NoDerivs 4.0 International License (CC BY-NC-ND 4.0), which permits the noncommercial replication and distribution of the article with the strict proviso that no changes or edits are made and the original work is properly cited (including links to both the formal publication through the relevant DOI and the license). See: https://creativecommons.org/licenses/by-nc-nd/4.0/.

\section{References}

1. Siegel RL, Miller KD, Jemal A. Cancer statistics, 2020. CA Cancer J Clin 2020;70:7-30.

2. Fitzmaurice C, Allen C, Barber RM, et al. Global, Regional, and National Cancer Incidence, Mortality, Years of Life Lost, Years Lived with Disability, and DisabilityAdjusted Life-years for 32 Cancer Groups, 1990 to 2015: A Systematic Analysis for the Global Burden of Disease Study. JAMA Oncol 2017;3:524-48.

3. Miller KD, Nogueira L, Mariotto AB, et al. Cancer treatment and survivorship statistics, 2019. CA Cancer J Clin 2019;69:363-85.

4. Ettinger DS, Wood DE, Aisner DL, et al. Non-Small Cell Lung Cancer, Version 5.2017, NCCN Clinical Practice Guidelines in Oncology. J Natl Compr Canc Netw 2017;15:504-35.

5. Paz-Ares L, Socinski MA, Shahidi J, et al. Correlation of 
EGFR-expression with safety and efficacy outcomes in SQUIRE: a randomized, multicenter, open-label, phase III study of gemcitabine-cisplatin plus necitumumab versus gemcitabine-cisplatin alone in the first-line treatment of patients with stage IV squamous non-small-cell lung cancer. Ann Oncol 2016;27:1573-9.

6. Pirker R. Conquering lung cancer: current status and prospects for the future. Pulmonology 2020;26:283-90.

7. Davies J, Patel M, Gridelli C, et al. Real-word treatment patterns for patients receiving second-line and thirdline treatment for advanced non-small cell lung cancer: a systematic review of recently published studies. PLoS One 2017;12:e0175679.

8. Tanaka I, Morise M, Miyazawa A, et al. Potential benefits of Bevacizumab combined with platinumbased chemotherapy in advanced non-small-cell lung cancer patients with EGFR mutation. Clin Lung Cancer 2020;21:273-280.e4.

9. Dienstmann R, Tabernero J. Necitumumab, a fully human IgG1 mAb directed against the EGFR for the potential treatment of cancer. Curr Opin Investig Drugs 2010;11:1434-41.

10. Bagchi A, Haidar JN, Eastman SW, et al. Molecular Basis for Necitumumab Inhibition of EGFR Variants Associated with Acquired Cetuximab Resistance. Mol Cancer Ther 2018;17:521-31.

11. U.S. Food and Drug Administration. FDA approves Portrazza to treat advanced squamous nonesmall-cell lung cancer. Available online: https://www.fda.gov/ drugs/new-drugs-fda-cders-new-molecular-entitiesand-new-therapeutic-biological-products/novel-drugapprovals-2015.

12. Thatcher N, Hirsch FR, Luft AV, et al. Necitumumab plus gemcitabine and cisplatin versus gemcitabine and cisplatin alone as first-line therapy in patients with stage IV squamous non-small-cell lung cancer (SQUIRE): an open-label, randomized, controlled phase 3 trial. Lancet Oncol 2015;16:763-74.

13. Watanabe S, Yoshioka H, Sakai H, et al. Corrigendum to "Necitumumab plus gemcitabine and cisplatin versus gemcitabine and cisplatin alone as first-line treatment for stage IV squamous non-small cell lung cancer: A phase $1 \mathrm{~b}$ and randomized, open-label, multicenter, phase 2 trial in Japan”. Lung Cancer 2019;132:157-8.

14. Watanabe S, Yoshioka H, Sakai H, et al. Necitumumab plus gemcitabine and cisplatin versus gemcitabine and cisplatin alone as first-line treatment for stage IV squamous non-small cell lung cancer: A phase lb and randomized, open-label, multicenter, phase 2 trial in Japan. Lung Cancer 2019;129:55-62.

15. Paz-Ares L, Mezger J, Ciuleanu TE, et al. Necitumumab plus pemetrexed and cisplatin as first-line therapy in patients with stage IV non-squamous non-small-cell lung cancer (INSPIRE): an open-label, randomized, controlled phase 3 study. Lancet Oncol 2015;16:328-37.

16. Spigel DR, Luft A, Depenbrock H, et al. An Open-Label, Randomized, Controlled Phase II Study of PaclitaxelCarboplatin Chemotherapy with Necitumumab Versus Paclitaxel-Carboplatin Alone in First-Line Treatment of Patients with Stage IV Squamous Non-Small-Cell Lung Cancer. Clin Lung Cancer 2017;18:480-8.

17. Edge SB, Compton CC. The American Joint Committee on Cancer: the 7th edition of the AJCC cancer staging manual and the future of TNM. Ann Surg Oncol 2010;17:1471-4.

18. Jadad AR, Moore RA, Carroll D, et al. Assessing the quality of reports of randomized clinical trials: is blinding necessary? Control Clin Trials 1996;17:1-12.

19. Guyatt GH, Oxman AD, Schünemann HJ, et al. GRADE guidelines: a new series of articles in the Journal of Clinical Epidemiology. J Clin Epidemiol 2011;64:380-2.

20. Higgins JP, Altman DG, Gøtzsche PC, et al. The Cochrane Collaboration's tool for assessing risk of bias in randomised trials. BMJ 2011;343:d5928.

21. Reck M, Socinski MA, Luft A, et al. The Effect of Necitumumab in Combination with Gemcitabine plus Cisplatin on Tolerability and on Quality of Life: Results from the Phase 3 SQUIRE Trial. J Thorac Oncol 2016;11:808-18.

22. Reck M, Thomas M, Kropf-Sanchen C, et al. Necitumumab plus Gemcitabine and Cisplatin as FirstLine Therapy in Patients with Stage IV EGFR- Expressing Squamous Non-Small-Cell Lung Cancer: German Subgroup Data from an Open-Label, Randomized Controlled Phase 3 Study (SQUIRE). Oncol Res Treat 2016;39:539-47.

23. Park K, Cho EK, Bello M, et al. Efficacy and Safety of First-Line Necitumumab Plus Gemcitabine and Cisplatin Versus Gemcitabine and Cisplatin in East Asian Patients with Stage IV Squamous Non-Small Cell Lung Cancer: A Subgroup Analysis of the Phase 3, OpenLabel, Randomized SQUIRE Study. Cancer Res Treat 2017;49:937-46.

24. Besse B, Adjei A, Baas P, et al. 2nd ESMO Consensus Conference on Lung Cancer: non-small-cell lung cancer first-line/second and further lines of treatment in advanced 
disease. Ann Oncol 2014;25:1475-84.

25. Zhou F, Zhou CC. Necitumumab for patients with nonsquamous NSCLC: uninspiring results. Lancet Oncol 2015;16:246-7.

26. Genova C, Varella-Garcia M, Rivard C, et al. EGFR Gene Copy Number by FISH May Predict Outcome of Necitumumab in Squamous Lung Carcinomas: Analysis from the SQUIRE Study. J Thorac Oncol 2018;13:228-36.

27. Petersen I, Dietel M, Geilenkeuser WJ, et al. EGFR immunohistochemistry as biomarker for antibody-based therapy of squamous NSCLC-Experience from the first

Cite this article as: Wang L, Liao C, Li M, Zhang S, Yi F, Wei Y, Yu J, Zhang W. Necitumumab plus platinum-based chemotherapy versus chemotherapy alone as first-line treatment for stage IV non-small cell lung cancer: a meta-analysis based on randomized controlled trials. Ann Palliat Med 2021;10(2):11541166. doi: 10.21037/apm-19-365 ring trial of the German Quality Assurance Initiative for Pathology (QuIP®). Pathol Res Pract 2017;213:1530-5.

28. Chigutsa E, Long AJ, Wallin JE. Exposure-response analysis of necitumumab efficacy in squamous non-small cell lung cancer patients. CPT Pharmacometrics Syst Pharmacol 2017;6:560-8.

29. Zugazagoitia J, Ponce S, Paz-Ares L. Necitumumab for first-line treatment of advanced, squamous, non-small-cell lung cancer: a relevant step forward. Transl Lung Cancer Res 2016;5:95-7. 
The combined text and medical subject heading (MeSH) terms used were: "lung cancer", "necitumumab" and "chemotherapy".

\section{PubMed}

The database was searched on May 20, 2019, N=53.

Search Strategy:

(Necitumumab [Title/Abstract] OR Portrazza [Title/Abstract]) OR IMC-11F8 [Title/Abstract] OR IMC-11F8 monoclonal antibody [Title/Abstract] )AND (pulmonary neoplasms [Title/Abstract] OR lung neoplasm [Title/Abstract] OR pulmonary neoplasm [Title/Abstract] OR lung cancer [title/Abstract] OR lung cancers [Title/Abstract] OR pulmonary cancer [Title/ Abstract] OR pulmonary cancers [Title/Abstract] OR cancer of the lung [Title/Abstract] OR cancer of lung [Title/ Abstract] OR NSCLC [Title/Abstract] OR Lung carcinoma [Title/Abstract] )AND (chemotherapy [Title/Abstract] OR drug chemotherapy [Title/Abstract] OR chemotherapies [Title/Abstract] OR pharmacotherapy [Title/Abstract] OR pharmacotherapies [Title/Abstract]).

\section{Scopus}

The database was searched on May 20, 2019, N=219.

Search Strategy:

(TITLE-ABS-KEY ("necitumumab" OR "Portrazza" OR "IMC-11F8" OR "IMC-11F8 monoclonal antibody") AND TITLE-ABS-KEY("pulmonary neoplasms" OR "lung neoplasm” OR "pulmonary neoplasm” OR "lung cancer" OR "lung cancers" OR "pulmonary cancer" OR "pulmonary cancers" OR "cancer of the lung" OR "cancer of lung" OR "NSCLC" OR "Lung carcinoma") AND TITLE-ABS-KEY(“drug therapies" OR "chemotherapy" OR "chemotherapies" OR "pharmacotherapy" OR "pharmacotherapies")).

\section{Web of Science}

The database was searched on May 20, 2019, N=83

Search Strategy:

\#1: TS=("pulmonary neoplasms" OR "lung neoplasm" OR "pulmonary neoplasm"

OR "lung cancer" OR "lung cancers" OR "pulmonary cancer" OR "pulmonary cancers" OR "cancer of the lung" OR "cancer of lung" OR "NSCLC" OR "Lung carcinoma") N=294444

\#2: TS=("Necitumumab" OR "PORTRAZZA" OR "IMC-11F8" OR "IMC-11F8 monoclonal antibody") N=121

\#3: TS=("drug therapies" OR "chemotherapy" OR "chemotherapies" OR "pharmacotherapy" OR "pharmacotherapies") $\mathrm{N}=1542150$

\#4: \#1 AND \#2 AND \#3 N=83

\section{Embase}

The database was searched on May 20, 2019, N=99

Search Strategy:

("Necitumumab" OR "PORTRAZZA" OR "IM-11F8" OR "IMC-11F8 monoclonal antibody"): ti, ab, kw AND ("pulmonary neoplasms" OR "lung neoplasm" OR "pulmonary neoplasm" OR "lung cancer" OR "lung cancers" OR "pulmonary cancer" OR "pulmonary cancers" OR "cancer of the lung" OR "cancer of lung" OR "NSCLC"): ti, ab, kw AND ("drug therapies" OR "chemotherapy" OR “chemotherapies" OR "pharmacotherapy" OR "pharmacotherapies”) :ti, ab, kw.

\section{Ovid}

The database was searched on May 20, 2019, N=262 
Search Strategy:

\#1: Necitumumab

\#2: Portrazza

\#3: IMC-11F8

\#4: IMC-11F8 monoclonal antibody

\#5: pulmonary neoplasms

\#6: pulmonary cancer

\#7: pulmonary cancers

\#8: cancer of the lung

\#9: cancer of lung

\#10: Lung carcinoma

\#11: Lung neoplasm

\#12: NSCLC

\#13: drug therapies

\#14: chemotherapy

\#15: chemotherapies

\#16: pharmacotherapy

\#17: pharmacotherapies

$\# 18: \# 1$ and \#2 and \#3 and \#4 and \#5 and \#6 and \#7 and \#8 and \#9 and \#10 and \#11 and \#12 and \#13 and \#14 and \#15 and \#16 and \#17

\section{Cochrane}

The database was searched on May 20, 2019, N=54

Search Strategy:

("Necitumumab" OR "Portrazza" OR "IMC-11F8" OR "IMC-11F8 monoclonal antibody"): ti, ab, kw AND ("pulmonary neoplasms" OR "lung neoplasm" OR "pulmonary neoplasm" OR "lung cancer" OR "lung cancers" OR "pulmonary cancer" OR "pulmonary cancers" OR "cancer of the lung" OR "cancer of lung" OR "NSCLC"): ti, ab, kw AND ("drug therapies" OR "chemotherapy" OR "chemotherapies" OR "pharmacotherapy" OR "pharmacotherapies") : ti, ab, kw. 
Table S1 GRADE quality assessment for the outcomes of survival, response rate and toxicity

\begin{tabular}{|c|c|c|c|c|c|c|c|c|c|}
\hline \multirow[b]{2}{*}{ Primary outcome } & \multirow[b]{2}{*}{ No. } & \multicolumn{2}{|c|}{ No. of participants } & \multirow{2}{*}{$\begin{array}{l}\text { Differences }^{a} \\
(95 \% \mathrm{Cl})\end{array}$} & \multicolumn{4}{|c|}{ Quality assessment } & \multirow[b]{2}{*}{ Quality } \\
\hline & & NC & $\mathrm{CA}$ & & Risk of bias ${ }^{b}$ & Inconsistency & Indirectness & $\begin{array}{c}\text { Publication } \\
\text { bias }^{c}\end{array}$ & \\
\hline \multicolumn{10}{|l|}{ Survival } \\
\hline OS & 4 & 1,060 & 1,014 & $0.93(0.85-1.01)$ & Low & No inconsistency & No indirectness No imprecision & Unlikely & High \\
\hline \multicolumn{10}{|l|}{ OSR } \\
\hline 0.5-year & 4 & $768 / 1,060$ & $712 / 1,014$ & $1.04(0.94-1.16)$ & Low & No inconsistency & No indirectness No imprecision & Unlikely & High \\
\hline 1-year & 4 & $490 / 1,060$ & $429 / 1,014$ & $1.13(0.94-1.36)$ & Low & No inconsistency & No indirectness No imprecision & Unlikely & High \\
\hline 1.5-year & 4 & $252 / 1,060$ & $232 / 1,014$ & $1.07(0.92-1.25)$ & Low & No inconsistency & No indirectness No imprecision & Unlikely & High \\
\hline 2-year & 3 & $116 / 950$ & $104 / 957$ & $1.24(0.70-2.17)$ & Serious $(-1)$ & No inconsistency & No indirectness No imprecision & Unlikely & Medium \\
\hline PFS & 4 & 1,060 & 1,014 & $0.91(0.84-0.99)$ & Low & No inconsistency & No indirectness No imprecision & Unlikely & High \\
\hline \multicolumn{10}{|l|}{ PFSR } \\
\hline 0.5-year & 4 & $354 / 1,060$ & $277 / 1,014$ & $1.22(0.88-1.69)$ & Low & No inconsistency & No indirectness No imprecision & Unlikely & High \\
\hline 1-year & 4 & $93 / 1,060$ & $69 / 1,014$ & $1.28(0.95-1.73)$ & Low & No inconsistency & No indirectness No imprecision & Unlikely & High \\
\hline 1.5-year & 3 & $39 / 1,060$ & $29 / 1,014$ & $1.35(0.84-2.15)$ & Low & No inconsistency & No indirectness No imprecision & Unlikely & High \\
\hline 2-year & 2 & $14 / 860$ & $7 / 866$ & $2.01(0.82-4.97)$ & Low & No inconsistency & No indirectness No imprecision & Unlikely & High \\
\hline \multicolumn{10}{|l|}{ Response rate } \\
\hline CR & 3 & $116 / 1,063$ & $8 / 1,014$ & $0.14(0.02-0.79)$ & Low & Serious $(-1)$ & No indirectness No imprecision & Unlikely & Medium \\
\hline PR & 4 & $116 / 1,064$ & $291 / 1,014$ & $1.17(1.03-1.33)$ & Low & No inconsistency & No indirectness No imprecision & Unlikely & High \\
\hline SD & 4 & $116 / 1,065$ & $473 / 1,014$ & $0.99(0.90-1.09)$ & Low & No inconsistency & No indirectness No imprecision & Unlikely & High \\
\hline PD & 4 & $116 / 1,066$ & $121 / 1,014$ & $0.68(0.53-0.89)$ & Low & No inconsistency & No indirectness No imprecision & Unlikely & High \\
\hline ORR & 4 & $116 / 1,061$ & $299 / 1,014$ & $1.26(0.93-1.71)$ & Low & No inconsistency & No indirectness No imprecision & Unlikely & High \\
\hline DCR & 4 & $116 / 1,062$ & $772 / 1,014$ & $1.05(1.00-1.10)$ & Low & No inconsistency & No indirectness No imprecision & Unlikely & High \\
\hline \multicolumn{10}{|l|}{ Toxicity } \\
\hline Total AEs & 2 & $324 / 425$ & $236 / 375$ & $1.12(0.97-1.31)$ & Low & No inconsistency & No indirectness No imprecision & Unlikely & High \\
\hline Grade 3-5 AEs & 3 & $612 / 963$ & $498 / 916$ & $1.14(1.01-1.28)$ & Low & No inconsistency & No indirectness No imprecision & Unlikely & High \\
\hline Dose reduction & 2 & $465 / 853$ & $410 / 853$ & $0.07(0.02-0.11)$ & Low & No inconsistency & No indirectness No imprecision & Unlikely & High \\
\hline $\begin{array}{l}\text { Dose } \\
\text { discontinuation }\end{array}$ & 3 & $262 / 963$ & $194 / 910$ & $1.30(1.11-1.53)$ & Low & No inconsistency & No indirectness No imprecision & Unlikely & High \\
\hline
\end{tabular}

a , differences: hazard ratio (HR) for OS and PFS; risk ratios (RR) for OSR, PFSR, CR, PR, SD, PD, ORR, DCR, total AEs, grade 3-5 AEs, dose reduction and dose discontinuation; ${ }^{b}$, risk of bias assessed using the Jadad Scale (NOS) for randomized controlled trials; ${ }^{c}$, publication bias was assessed by Egger's and Begg's tests. OS, overall survival; OSR, overall survival rate; PFS, progression free survival; PFSR, progression free survival rate; NC, necitumumab plus platinum-based chemotherapy; CA, platinum-based chemotherapy alone; $\mathrm{Cl}$, confidence interval. 
A

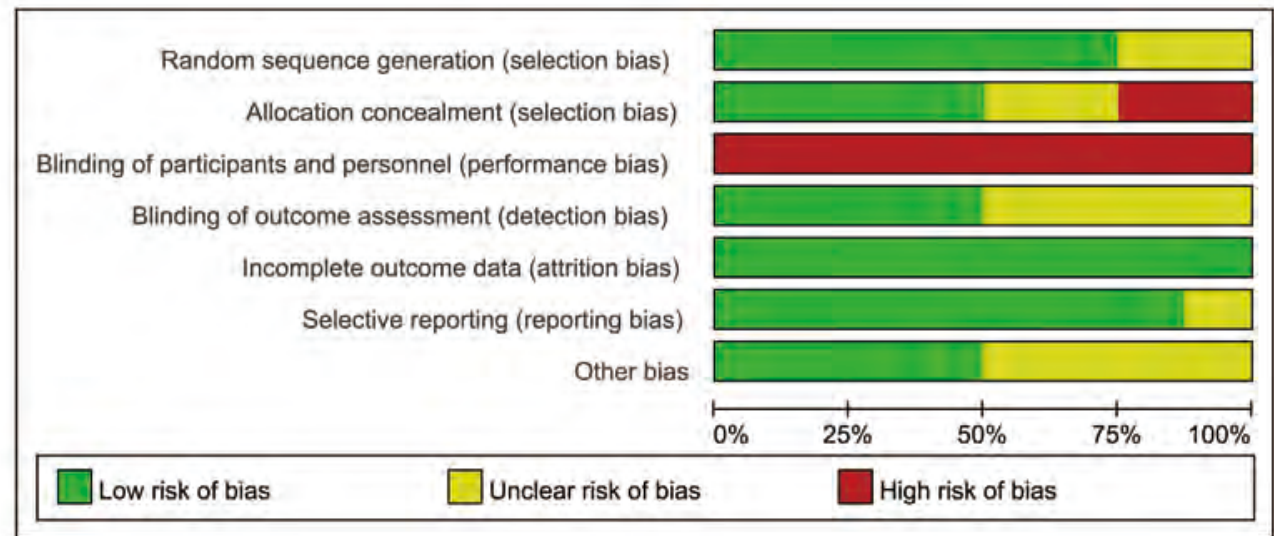

B

\begin{tabular}{|c|c|c|c|c|c|c|c|c|}
\hline 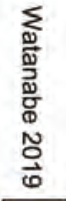 & 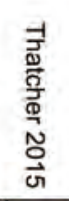 & $\begin{array}{l}\text { OD } \\
0.0 \\
\frac{0}{0} \\
N \\
\stackrel{0}{V}\end{array}$ & 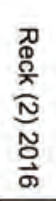 & 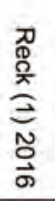 & 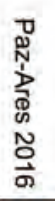 & 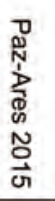 & 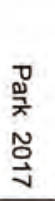 & \\
\hline$\oplus$ & $\oplus$ & $\oplus$ & $\oplus$ & $\oplus$ & 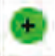 & $\sim$ & $\sim$ & Random sequence generation (selection bias) \\
\hline$\odot$ & $\oplus$ & $\oplus$ & $\sim$ & $\omega$ & $\oplus$ & (1) & (1) & Allocation concealment (selection bias) \\
\hline (1) & (1) & (1) & (1) & (1) & (1) & (1) & ( & Blinding of participants and personnel (performance bias) \\
\hline$\sim$ & $\omega$ & $\sim$ & $\oplus$ & $\oplus$ & $\sim$ & $\oplus$ & $\oplus$ & Blinding of outcome assessment (detection bias) \\
\hline$\oplus$ & $\oplus$ & 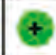 & $\oplus$ & $\oplus$ & $\hookrightarrow$ & $\oplus$ & $\oplus$ & Incomplete outcome data (attrition bias) \\
\hline$\oplus$ & $\oplus$ & $\sim$ & $\oplus$ & $\oplus$ & $\odot$ & $\oplus$ & $\oplus$ & Selective reporting (reporting bias) \\
\hline$\sim$ & $\oplus$ & $\oplus$ & $\sim$ & $\omega$ & $\sim$ & 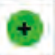 & $\oplus$ & Other bias \\
\hline
\end{tabular}

Figure S1 Cochrane Risk Assessment associated with necitumumab plus platinum-based chemotherapy versus platinum-based chemotherapy alone. 


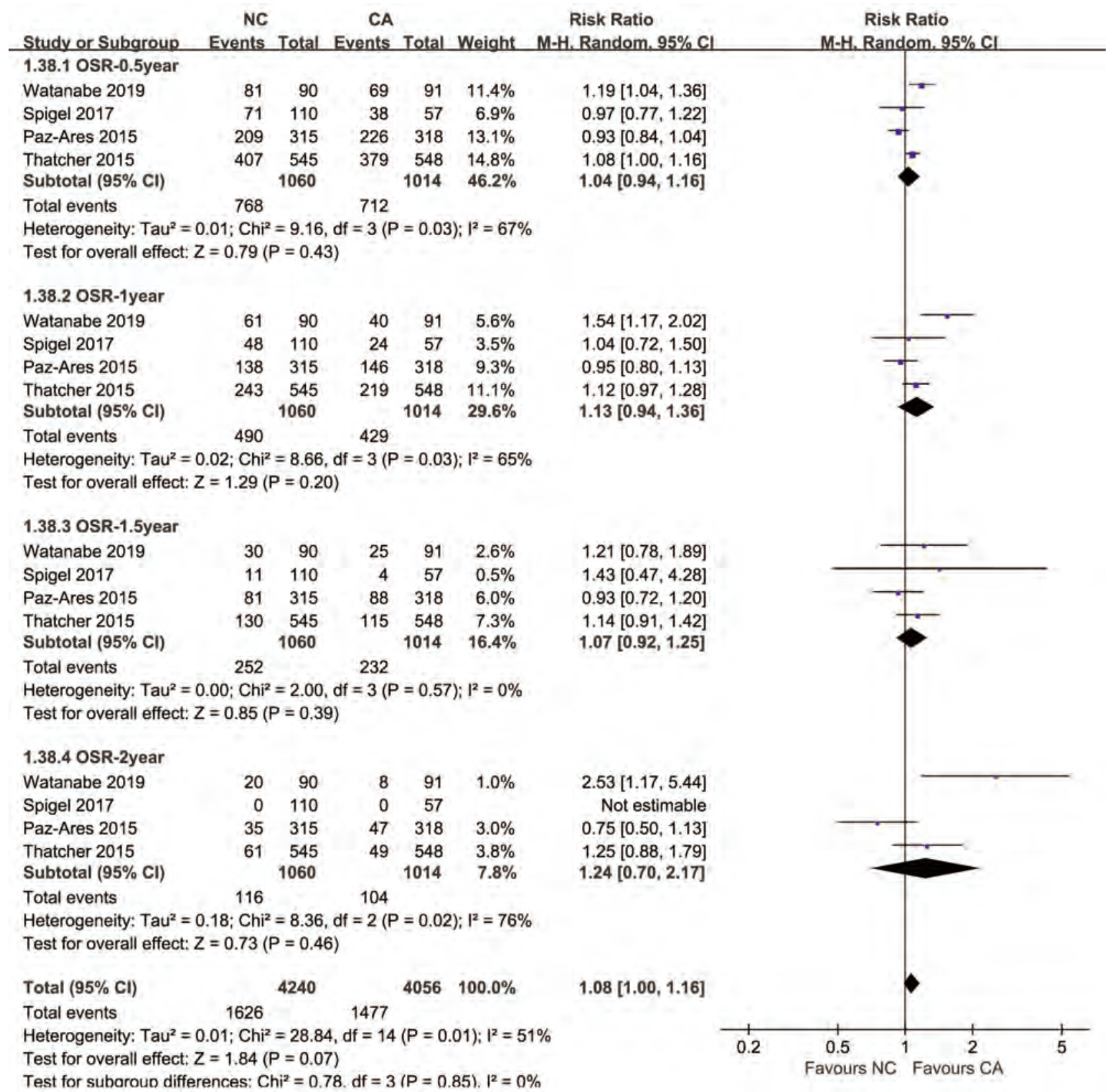

Figure S2 Forest plots of OSR-0.5y, OSR-1y, OSR-1.5y and OSR-2y associated with necitumumab plus platinum-based chemotherapy versus platinum-based chemotherapy alone. OSR, overall survival rate. 


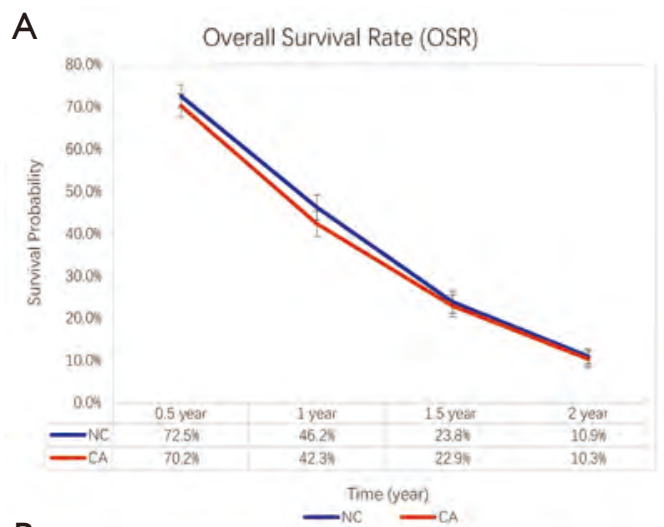

B

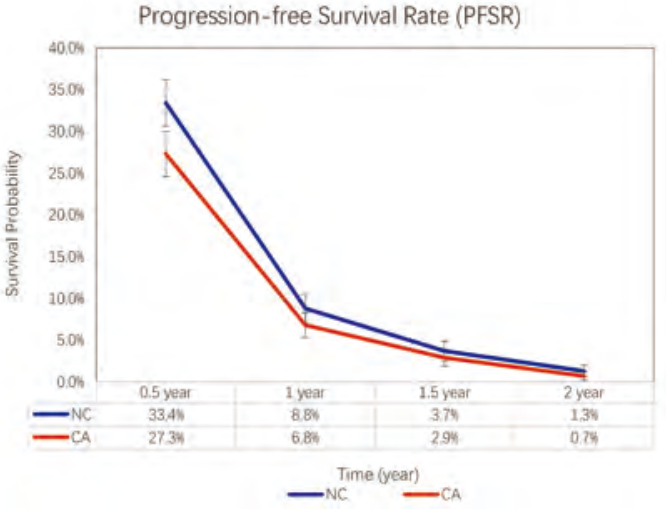

Figure S3 Trends in the RR of OSR (A) and PFSR (B) over time associated with necitumumab plus platinum-based chemotherapy versus platinum-based chemotherapy alone. RR, risk ratios; OSR, overall survival rate; PFSR, progression free survival rate. 


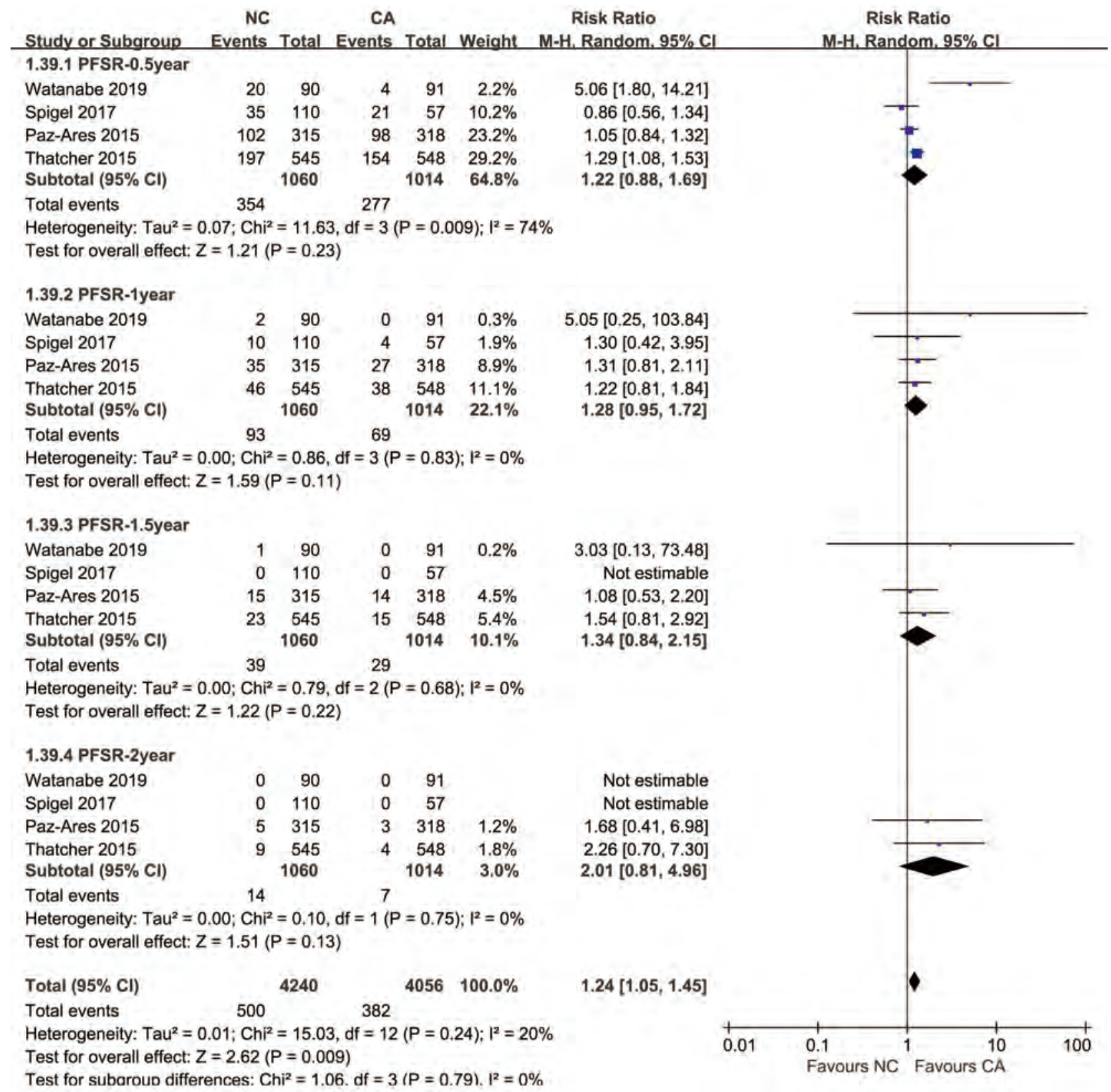

Figure S4 Forest plots of PFSR-0.5y, PFSR-1y, PFSR-1.5y and PFSR-2y associated with necitumumab plus platinum-based chemotherapy versus platinum-based chemotherapy alone. PFSR, progression free survival rate. 
A

\begin{tabular}{|c|c|c|c|c|c|c|c|c|c|c|}
\hline Study or Subgroup & $\begin{array}{r}\text { NC } \\
\text { Events }\end{array}$ & Total & $\begin{array}{r}\text { CA } \\
\text { Events }\end{array}$ & Total & Weight & $\begin{array}{c}\text { Risk Ratio } \\
\text { M-H. Fixed. } 95 \% \mathrm{Cl}\end{array}$ & \multicolumn{4}{|c|}{$\begin{array}{c}\text { Risk Ratio } \\
\text { M-H. Fixed. } 95 \% \mathrm{Cl}\end{array}$} \\
\hline Spigel 2017 & 0 & 110 & 1 & 57 & $7 \quad 19.8 \%$ & $0.17[0.01,4.21]$ & & & & \\
\hline Paz-Ares 2015 & 0 & 315 & 4 & 318 & $8 \quad 45.1 \%$ & $0.11[0.01,2.07]$ & & & & \\
\hline Thatcher 2015 & 0 & 538 & 3 & 541 & $1 \quad 35.1 \%$ & $0.14[0.01,2.77]$ & & & & \\
\hline Total $(95 \% \mathrm{Cl})$ & & 963 & & 916 & $6 \quad 100.0 \%$ & $0.14[0.02,0.79]$ & & & & \\
\hline Total events & 0 & & 8 & & & & & & & \\
\hline \multicolumn{7}{|c|}{$\begin{array}{l}\text { Heterogeneity: } \mathrm{Chi}^{2}=0.04, \mathrm{df}=2(P=0.98) ; \mathrm{I}^{2}=0 \% \\
\text { Test for overall effect: } Z=2.23(P=0.03)\end{array}$} & 0.005 & Favours CA $^{0.1}$ & $\begin{array}{l}10 \\
\text { Favours NC }\end{array}$ & $\frac{1}{200}$ \\
\hline Study or Subgroup & $\begin{array}{r}\text { NC } \\
\text { Events }\end{array}$ & Total & $\begin{array}{r}\text { CA } \\
\text { Events }\end{array}$ & Total & Weight & $\begin{array}{c}\text { Risk Ratio } \\
\text { M-H. Random, } 95 \% \mathrm{CI}\end{array}$ & & $\begin{array}{r}\text { Risk } \\
\text { M-H. Rand }\end{array}$ & $\begin{array}{l}\text { Ratio } \\
\text { dom. } 95 \% \mathrm{Cl}\end{array}$ & \\
\hline Watanabe 2019 & 46 & 90 & 19 & 91 & $19.6 \%$ & $2.45[1.56,3.83]$ & & & 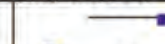 & \\
\hline Spigel 2017 & 46 & 110 & 19 & 57 & $20.3 \%$ & $1.25[0.82,1.93]$ & & & & \\
\hline Paz-Ares 2015 & 99 & 315 & 98 & 318 & $29.0 \%$ & $1.02[0.81,1.29]$ & & & & \\
\hline Thatcher 2015 & 170 & 538 & 155 & 541 & $31.1 \%$ & $1.10[0.92,1.32]$ & & & & \\
\hline Total $(95 \% \mathrm{Cl})$ & & 1053 & & 1007 & $100.0 \%$ & $1.29[0.96,1.74]$ & & & & \\
\hline Total events & 361 & & 291 & & & & & & & \\
\hline $\begin{array}{l}\text { Heterogeneity: Tau² = } \\
\text { Test for overall effect: }\end{array}$ & $\begin{array}{l}0.07 ; \mathrm{Chi}^{2} \\
\mathrm{z}=1.70(\mathrm{~F}\end{array}$ & $\begin{array}{l}=12.29 \\
=0.09\end{array}$ & , $d f=3(P$ & $P=0.00$ & $06) ; 1^{2}=76$ & $6 \%$ & 0.2 & $\begin{array}{l}0.5 \\
\text { Favours CA }\end{array}$ & $1 \frac{2}{\text { Favours }} \mathrm{NC}$ & 5 \\
\hline
\end{tabular}

C

\begin{tabular}{|c|c|c|c|c|c|c|c|c|c|c|}
\hline Study or Subgroup & $\begin{array}{r}N C \\
\text { Events }\end{array}$ & Total & $\begin{array}{r}\text { CA } \\
\text { Events }\end{array}$ & Total & Weight & $\begin{array}{c}\text { Risk Ratio } \\
\text { M-H. Random. } 95 \% \text { Cl }\end{array}$ & & $\begin{array}{r}\text { Risk } \\
\text { M-H. Ranc }\end{array}$ & $\begin{array}{l}\text { Ratio } \\
\text { dom. } 95 \% \mathrm{Cl}\end{array}$ & \\
\hline Watanabe 2019 & 38 & 90 & 54 & 91 & $18.7 \%$ & $0.71[0.53,0.96]$ & & - & & \\
\hline Spigel 2017 & 36 & 110 & 22 & 57 & $11.3 \%$ & $0.85[0.56,1.29]$ & & & & \\
\hline Paz-Ares 2015 & 136 & 315 & 133 & 318 & $30.7 \%$ & $1.03[0.86,1.24]$ & & & & \\
\hline Thatcher 2015 & 276 & 538 & 264 & 541 & $39.3 \%$ & $1.05[0.93,1.18]$ & & & & \\
\hline Total $(95 \% \mathrm{Cl})$ & & 1053 & & 1007 & $100.0 \%$ & $0.95[0.81,1.11]$ & & & & \\
\hline Total events & 486 & & 473 & & & & & & & \\
\hline \multicolumn{7}{|c|}{$\begin{array}{l}\text { Heterogeneity: } \mathrm{Tau}^{2}=0.01 ; \mathrm{Ch}^{2}=6.47, \mathrm{df}=3(P=0.09) ; \mathrm{I}^{2}=54 \% \\
\text { Test for overall effect: } Z=0.64(P=0.52)\end{array}$} & 0.5 & $\begin{array}{l}0.7 \\
\text { Favours CA }\end{array}$ & ${ }^{1}$ Favours NC & 2 \\
\hline
\end{tabular}

D

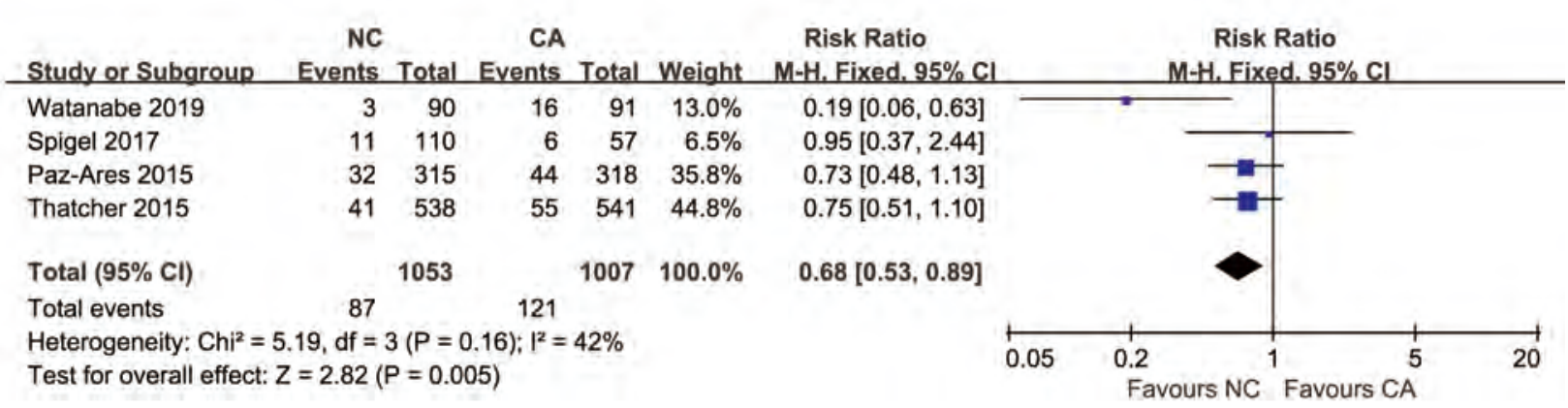

Figure S5 Forest plots of CR (A), PR (B), SD (C) and PD (D) associated with necitumumab plus platinum-based chemotherapy versus platinum-based chemotherapy alone. CR, complete remission; PR, partial remission; SD, stable disease; PD, progression of disease. 
A

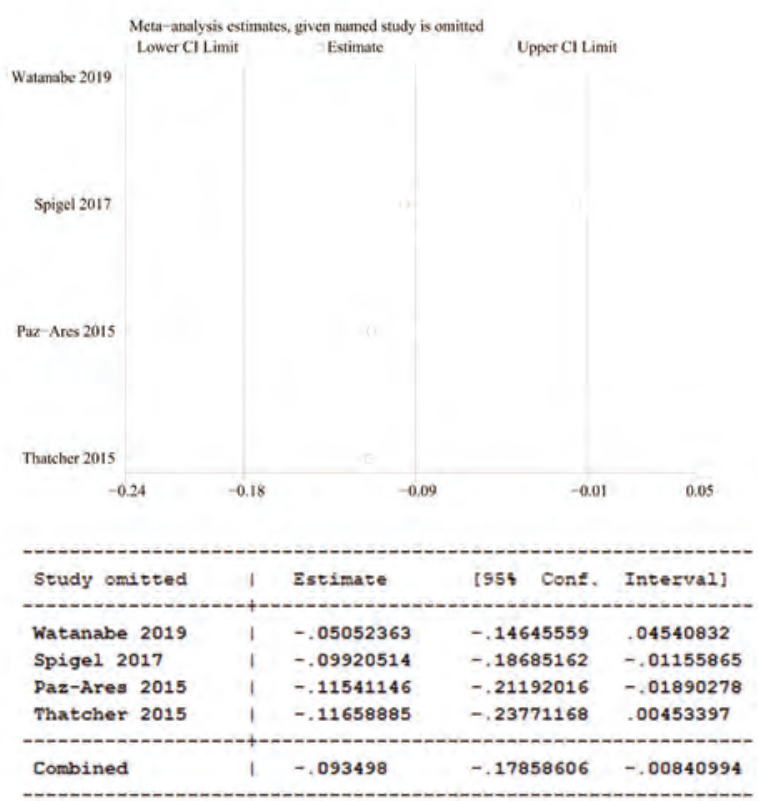

C

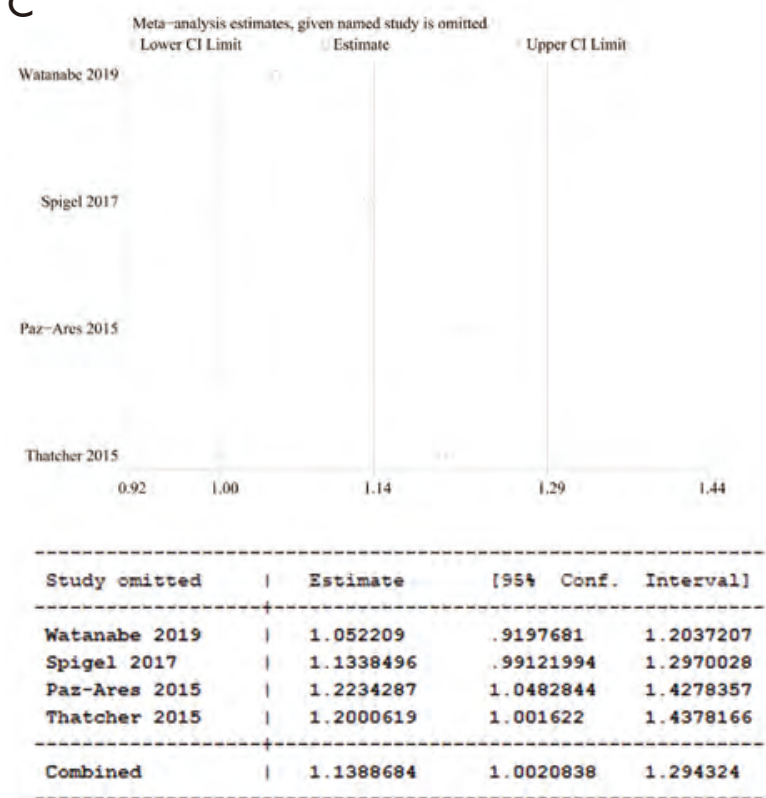

B

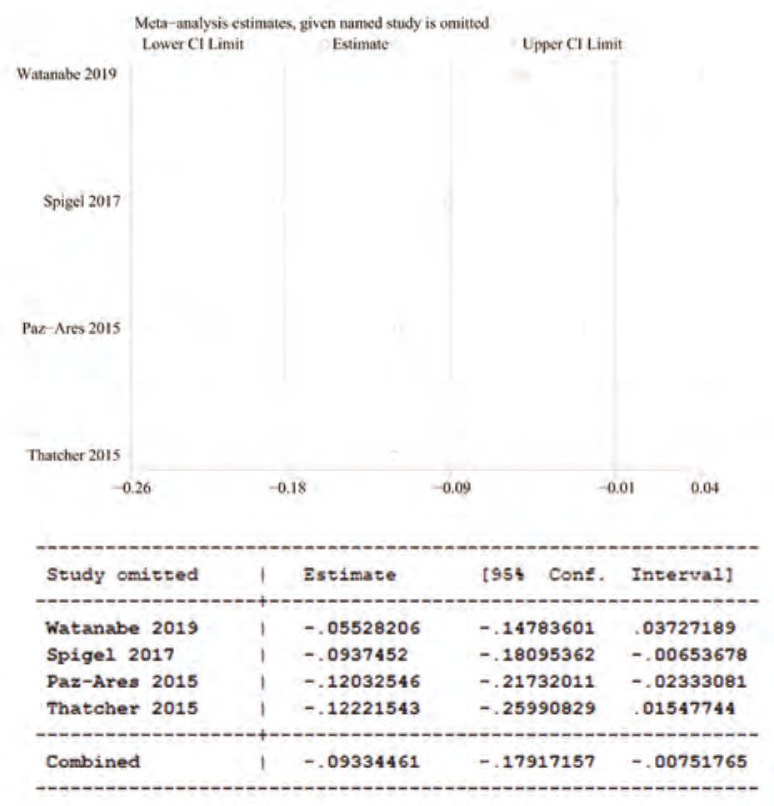

D

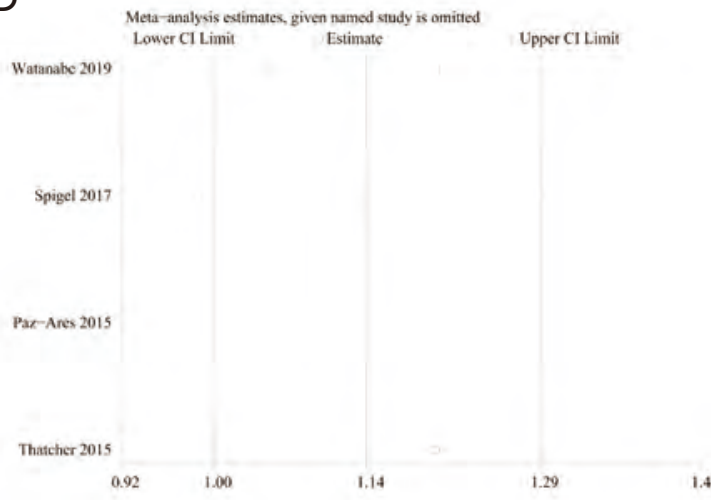

\begin{tabular}{l|llll} 
Study omitted & I & Estimate & [954 Conf. & Interval] \\
\hline Watanabe 2019 & I & 1.052209 & .9197681 & 1.2037207 \\
Spigel 2017 & I & 1.1338496 & .99121994 & 1.2970028 \\
Paz-Ares 2015 & I & 1.2234287 & 1.0482844 & 1.4278357 \\
Thatcher 2015 & I & 1.2000619 & 1.001622 & 1.4378166 \\
\hline Combined & 1 & 1.1388684 & 1.0020838 & 1.294324
\end{tabular}

Figure S6 Sensitivity analysis of PFS (A), OS (B), ORR (C) and DCR (D) associated with necitumumab plus platinum-based chemotherapy versus platinum-based chemotherapy alone. PFS, progression free survival; OS, overall survival; ORR, objective response rate; DCR, disease control rate. 
A

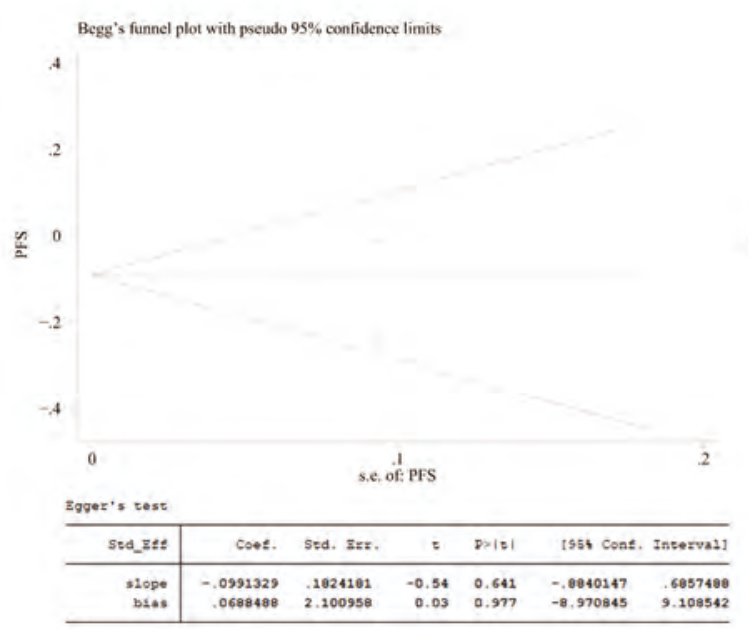

C

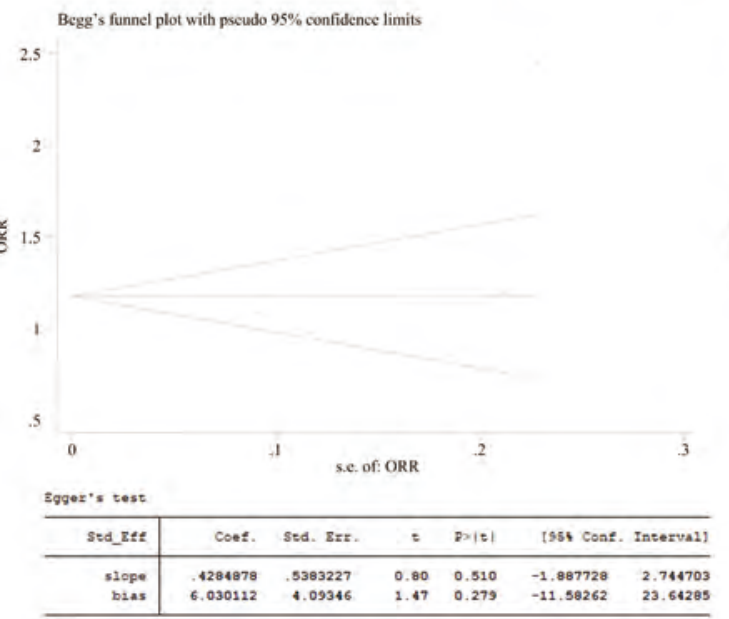

B

Begg's funnel plot with pseudo $95 \%$ confidence limits

s 0

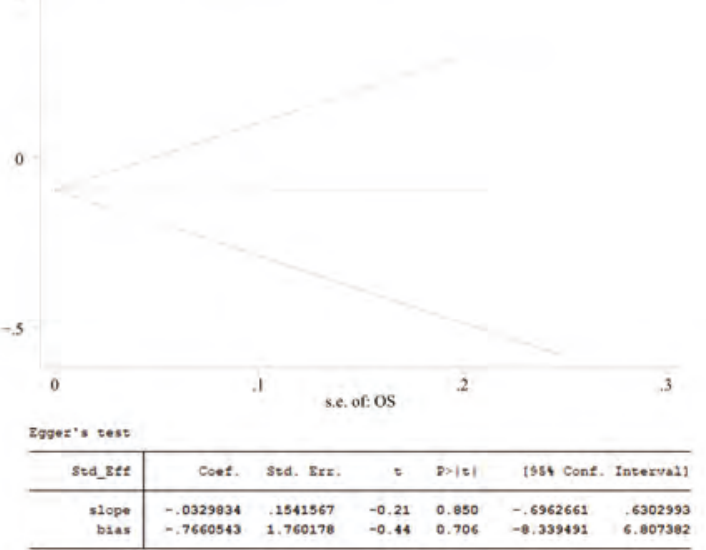

D

1.4

Begg's funnel plot with pseudo $95 \%$ confidence limits

1.2

\section{๕.}

8

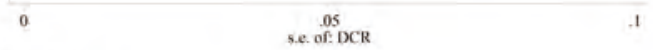

Egger $\times$ tsest

\begin{tabular}{|c|c|c|c|c|c|c|}
\hline SEd_zete & coes. & Std. Exs. & $=$ & $p>1=1$ & 1954 Cons. & Inserva1] \\
\hline slope & 1.055929 & 0927875 & 11,30 & 0.008 & .6566969 & 1. 455162 \\
\hline bias & .1469123 & 2.021603 & 0.07 & 0.949 & -8.551344 & 8. 845169 \\
\hline
\end{tabular}

Figure S7 The publication bias of PFS (A), OS (B), ORR (C) and DCR (D) associated with necitumumab plus platinum-based chemotherapy versus platinum-based chemotherapy alone. PFS, progression free survival; OS, overall survival; ORR, objective response rate; DCR, disease control rate. 